\title{
Synergy of Nano-ZnO and 3D-Graphene Foam Electrodes for Asymmetric Supercapacitor Devices
}

by:

Maryam Toufani

Submitted to the Graduate School of Engineering and Natural Sciences In partial fulfillment of the requirements for the degree of Master of Science

Sabanci University

August 2020 


\section{Synergy of Nano-ZnO and 3D-Graphene Foam Electrodes for Asymmetric Supercapacitor Devices}

APPROVED BY:

Assoc. Prof. Dr Emre Erdem (Thesis Supervisor)

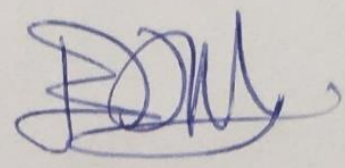

Assoc. Prof. Dr Meltem Sezen

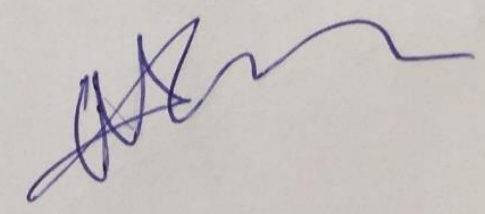

Prof. Dr Ebru Mensur-Alkoy

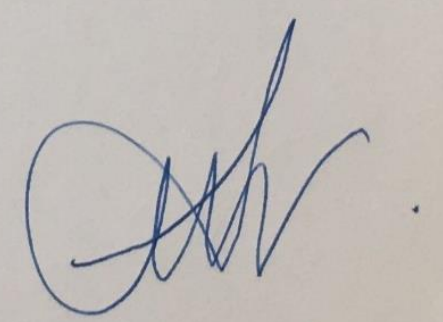

DATE OF APPROVAL: $11 / 08 / 2020$ 
(C) Maryam Toufani 2020

All Rights Reserved 


\title{
Synergy of Nano-ZnO and 3D-Graphene Foam Electrodes for Asymmetric Supercapacitor Devices
}

\author{
Maryam Toufani \\ MAT, Master Thesis, 2020 \\ Thesis Supervisor: Prof. Dr. Emre Erdem
}

Keywords: Supercapacitor, Zinc Oxide, Graphene Foam, Defect Structure

\begin{abstract}
Two kinds of electrode materials were produced to fabricate asymmetric supercapacitor devices: (i) Highly defective, n-type wide bandgap semiconductor $\mathrm{ZnO}$ nanocrystalline electrodes below $50 \mathrm{~nm}$ weresynthesized with the aid of the high energy ball milling technique. (ii) Flexible 3D-graphene foams weresynthesized via the chemical vapor deposition technique. Extensive defect structure analysis was performed via enhanced characterization techniques mainly the spectroscopy ones: electron paramagnetic resonance (EPR), Raman, and photoluminescence (PL). Compared to bulk $\mathrm{ZnO}$ electrodes the nanoscale $\mathrm{ZnO}$ electrodes revealed a dramatic increase of defect concentration. The surface defect plays a crucial role in the electrochemical performance of supercapacitor devices. Strong decreases in charge transfer resistance were observed for the smallest crystallite size which is $15 \mathrm{~nm}$. This work also shows that synthesis, controlling the defect structures, electronic and electrical characterization and the device production are extremely important to obtain high performance faradaic asymmetric supercapacitors.
\end{abstract}




\title{
Asimetrik Süper Kapasitör Cihazları İçin Nano-Zno Ve 3D-Grafen Köpük Elektrotlarının Sinerjisi
}

\author{
Maryam Toufani \\ MAT, Yüksek lisans Tezi, 2020 \\ Tez Danışmanı: Prof. Dr. Emre Erdem
}

Anahtar Kelimeler: süper kapasitör, Zinc Oxide, Graphene Foam, Kusur

\begin{abstract}
Özet
Asimetrik süper kapasitör cihazları üretebilmek için 2 tip elektrot malzemesi üretildi: (i) Yüksek enerjili bilyeli öğütme tekniğinin yardımıyla $50 \mathrm{~nm}$ altında, yüksek oranda kusurlu, n-tipi geniş bant aralıklı yarı iletken $\mathrm{ZnO}$ nanokristalin elektrotlar sentezlendi. (ii) Kimyasal buhar biriktirme tekniğiyle esnek 3Dgrafen köpüklersentezlendi. Kapsamlı kusur yapısı analizi, esasen spektroskopi olan gelişmiş karakterizasyon teknikleriyle gerçekleştirildi: elektron paramanyetik rezonans (EPR), Raman ve fotolüminesans (PL). Yığın $\mathrm{ZnO}$ elektrotlarına kıyasla nano ölçekli ZnO elektrotları, kusur konsantrasyonunda çarpıcı bir artış gösterdi. Yüzey kusuru, süper kapasitör cihazların elektrokimyasal performansında kritik bir rol oynar. En küçük kristalit boyutu olan $15 \mathrm{~nm}$ için yük transfer direncinde güçlü düşüşler gözlemlendi. Bu çalışma aynı zamanda sentezin, kusuryapılarının kontrolünün, elektronik ve elektriksel karakterizasyonun ve cihaz üretiminin yüksek performanslı faradaik asimetrik süper kapasitörler için son derece önemli olduğunu gösteriyor.
\end{abstract}




\section{Table of Contents}

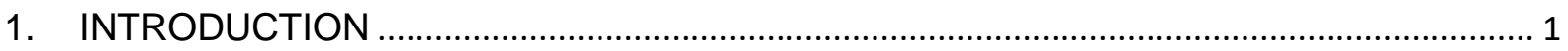

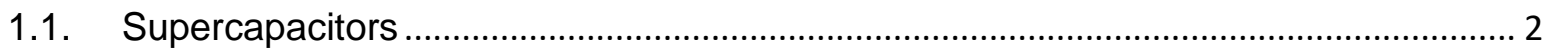

1.2. Fundamental and applications of supercapacitors supercapacitors........................... 3

1.2.1 Electrical double layer capacitor (EDLC) ….......................................................... 4

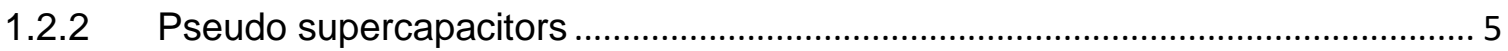

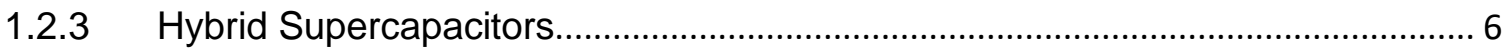

1.3 Structure of the Supercapacitors ............................................................................ 7

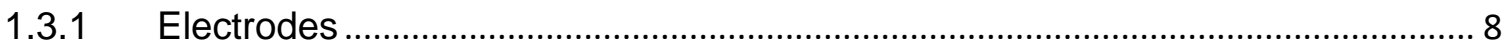

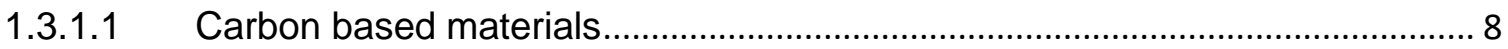

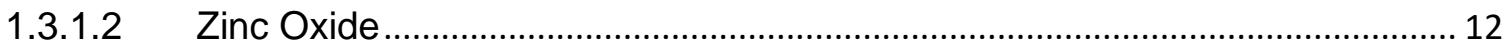

1.3.1.3 Defect structure of $\mathrm{ZnO}$ and Graphene ...................................................... 13

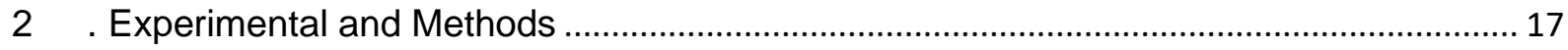

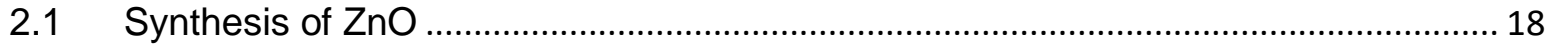

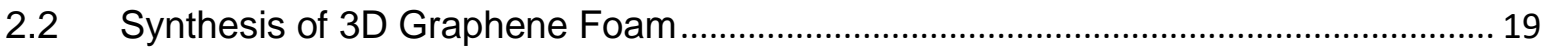

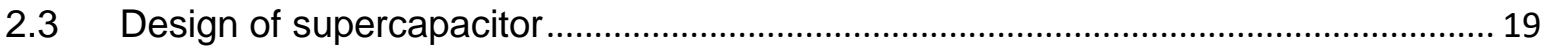

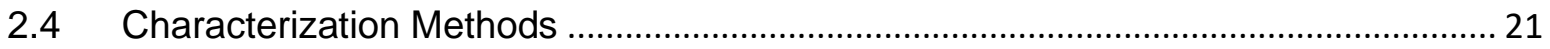

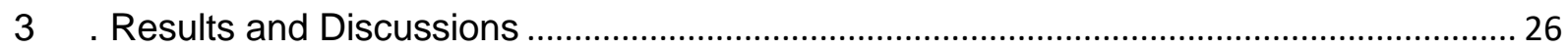

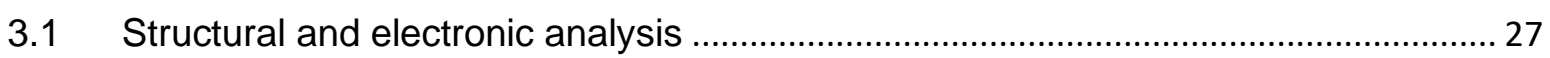

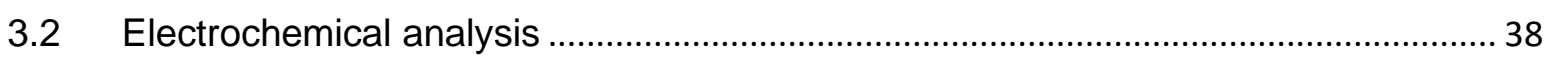

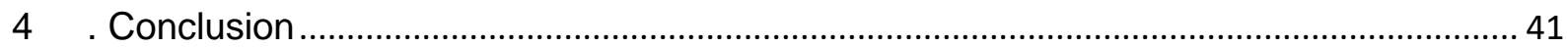




\section{List of Figures:}

Figure 1. Ragone plot which demonstrates energy vs. power density of supercapacitors with

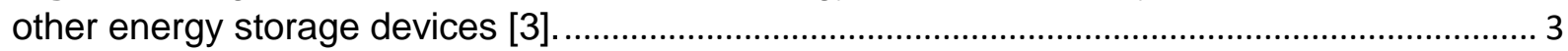

Figure 2. Classification of various types of supercapacitors......................................................... 4

Figure 3. Schematic of an electrical double layer capacitor [4] .............................................. 5

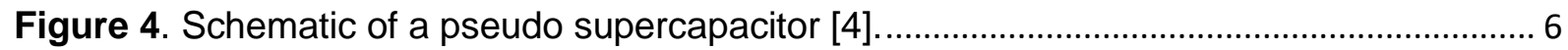

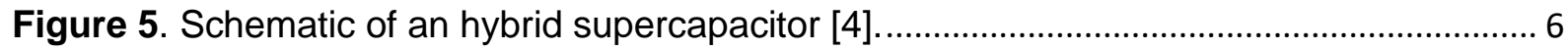

Figure 6. The general schematic of supercapaitor E1 and E2 are the electrodes and EL is

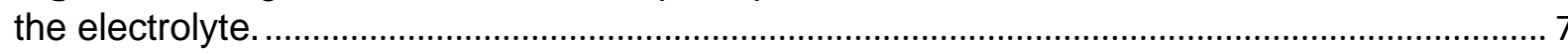

Figure 7. The Shematic of inside of the symmetric supercapacitor.......................................... 7

Figure 8. Different types of defects in Graphene: a) vacancy, b) divacancy, c) Stone-wale, d) adatom [12].

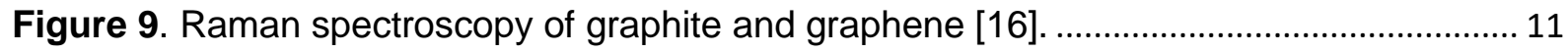

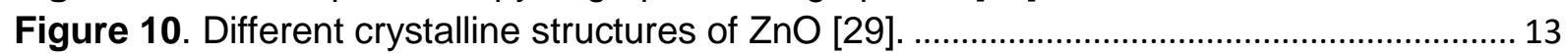

Figure 11. Representative EPR and PL spectra of defect structures in ZnO nanocrystal. In EPR only EPR active paramagnetic species can be observed whereas in PL all possible defect centers contributed to the defect-related visible emission band which is quite broad $(420 \mathrm{~nm}-700 \mathrm{~nm})$ [31].

Figure 12. Schematic of ball milling powder [49].

Figure 13. Graphical illustration of asymmetric supercapacitor model. The synergy of nanosized metal oxide $\mathrm{ZnO}$ and 3D-GF meets with the standard 1.0 M LiPF6 in $\mathrm{EC} / \mathrm{DEC}=50 / 50$ electrolyte deparated with glass fiber.

Figure 14. Incidence of electron beam on the sample and creation of secondary electrons, back scattered electron and characteristic $X$-rays [56].

Figure 15. a) XRD patterns of 3D-GF and HEBM ZnO nanocrystals. Different milling times result in different average sizes. b) HEBM holding time with respect to mean crystallite sizes obtained from XRD profiles. The figure revealed that with a very low mechanical e energy it is possible to reach from micron size down to a few tens of $n m$ size.

Figure 16. (a) SE-SEM images of 3D-GF with two different magnifications indicating porous structure. (b) Raman spectra of 3D-GF indicating $G$ and 2D bands. The defect-related D band is absent.

Figure 17. SEM images of HEBM ZnO nanocrystals indicating hexagonal structure. By decreasing average size to sub-30 nm, strong distortions occur from the hexagonal symmetry.

Figure 18. a) EPR spectra of HEBM ZnO nanoparticles and 3D-GF, b) absorption (integration of first derivative EPR spectra) of HEBM ZnO for 15 and $55 \mathrm{~nm}$ particle size. c) Photoluminescence spectra of HEBM ZnO nanoparticles (evolution of PL spectra with respect to crystalline size).

Figure 19. Size-dependent Raman spectra of HEBM ZnO crystals. The inset figure shows the region of $300-500 \mathrm{~cm}^{-1}$.

Figure 20. The electrochemical behavior of three produced asymmetric supercapacitors investigated by a) EIS in Nyquist plot of the hybrid supercapacitor, b) the variation of characteristic impedance (absolute value $|Z|$ ) with frequency (Bode-plot) and, c) behavior of intercalation-deintercalation (charge-discharge) via the cyclic voltammetry (CV). 


\section{List of Tables}

Table 1. Components of the various supercapacitors which are designed based on Fig. 13. Note that; LiPF6:1M lithium hexafluorophosphate dissolved in EC/DMC=50/50 (v/v).

Table 2. $g$-factor and the line width values of core and surface defects of HEBM ZnO nanocrystals obtained from X-band EPR spectra at room temperature.

Table 3. The concentrations of the defect centers obtained by using the spin-counting procedure [47].

Table 4. First- and second-order Raman spectra frequencies of $\mathrm{ZnO}$ nanoparticles obtained via HEBM.

Table 5. Examination of bandgap energies by UV-Vis spectroscopy of HEBM ZnO nanocrystals. The band-gap values were obtained by the aid of Tauc-plot. 
Chapter 1

\section{INTRODUCTION}




\subsection{Supercapacitors}

Nowadays, by increasing the depletion of fossil fuels and increasing pollution it is important to find a smart alternative to produce energy from other materials. One of the ways to overcome this issue is clean-energy such as rechargeable batteries, fuel cells, and supercapacitors. Among all mentioned, supercapacitors attracted much attention due to their significant properties. Mainly, they are used in hybrid electric vehicles such as automobiles, trains, and elevators since the rapid charge/ discharge cycles are necessary. Also, they are used widely in electronic and computer applications especially for memory protection and power back up [1]. In comparison to batteries, supercapacitors have high power density so, they can be used in applications that need high power with low energy density. Another important property of supercapacitors is that they can charge and discharge in a matter of seconds. The most important characteristic of supercapacitors is their high cycle life. They can tolerate many cycles by storing many electrons on the surface of electrodes on their double layer. The other feature of this device is its high performance even at - $40^{\circ} \mathrm{C}$ which is not achievable with batteries. Thus, supercapacitors are safer for high power charge and discharge applicants in comparison to batteries [2]. However, supercapacitors have low energy density and higher cost for their production which is an obstacle for this device. Fig. 1 is known as the Ragone plot. This plot is for comparision of energy density and power density for different devices.as it is obvious supercapacitors are placed among conventional capacitors and batteries. They have higher capacitances in comparison with conventional supercapacitors but as mentioned before they have lower energy density [3]. Therefore, the aim is to improve the properties of supercapacitors to find a way to enhance both power density and energy density. Finding the bests electrodes that can be used in supercapacitors is one way to improve these properties. Thus, criteria that should take into account for designing high-performance supercapacitors electrodes are as following: 1) high surface area: is leading to increase in specific capacitance of supercapacitors since charges are stored on the surface of electrodes. 2) electronic conductivity: the higher ionic and electronic conductivity can cause higher specific capacitance. Controlling pore sized electrodes can be helpful since it can cause more electron pathways to transfer. 3) mechanical and chemical stability: cyclic instability 
usually happens in supercapacitors due to the dissolution, side reactions, and phase changes in the electrodes. Using the type of electrodes with surface protection can make cyclic stability better [1].

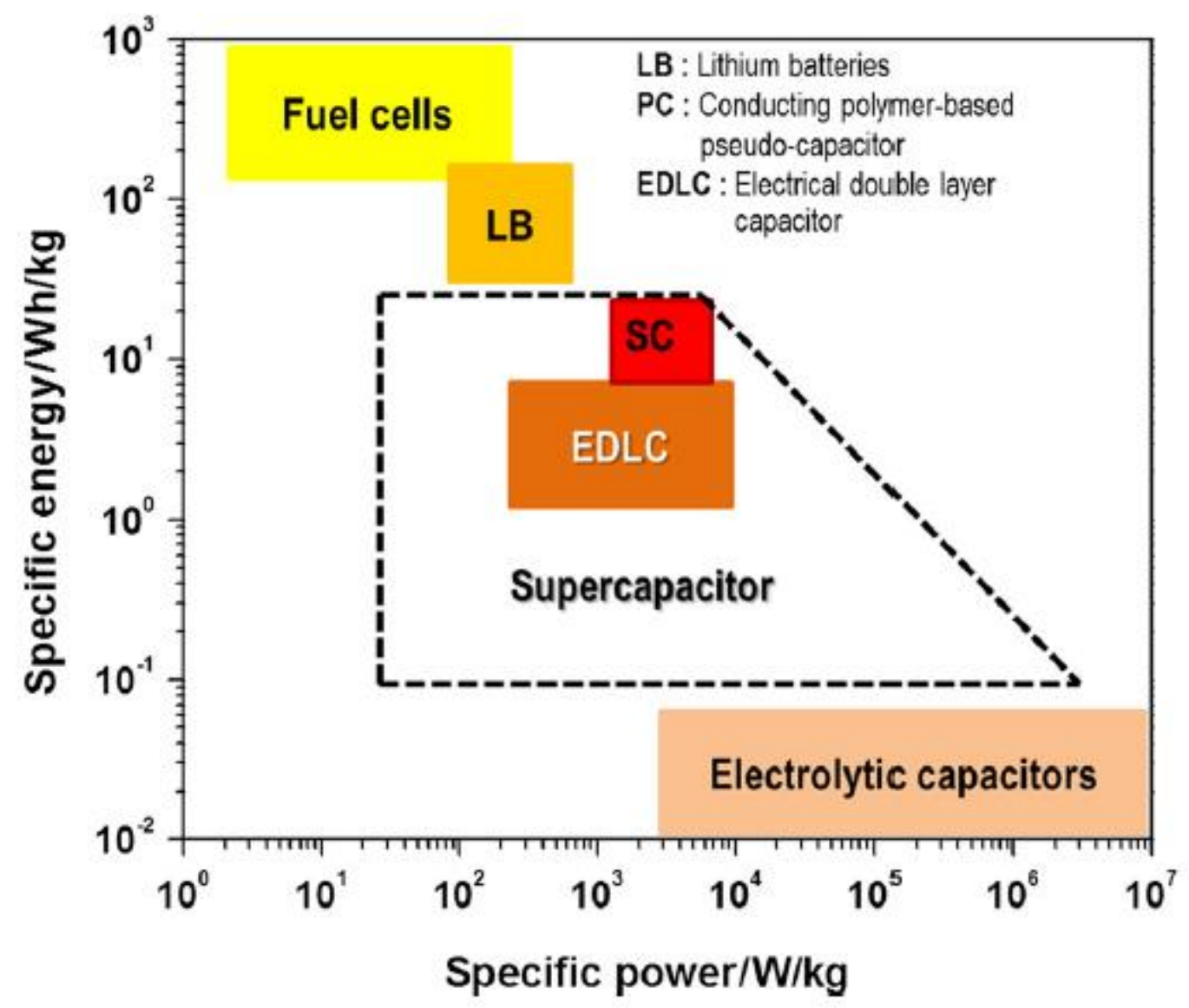

Figure 1. Ragone plot which demonstrates energy vs. power density of supercapacitors with other energy storage devices [3].

\subsection{Fundamental and applications of supercapacitors supercapacitors}

Normally, supercapacitors have three types: Electrical double layer supercapacitors (EDLS), pseudo supercapacitors and hybrid supercapacitors. Fig. 2 shows the different classificaiton of supercapacitors and the electrodes that are used in these supercapacitors. 


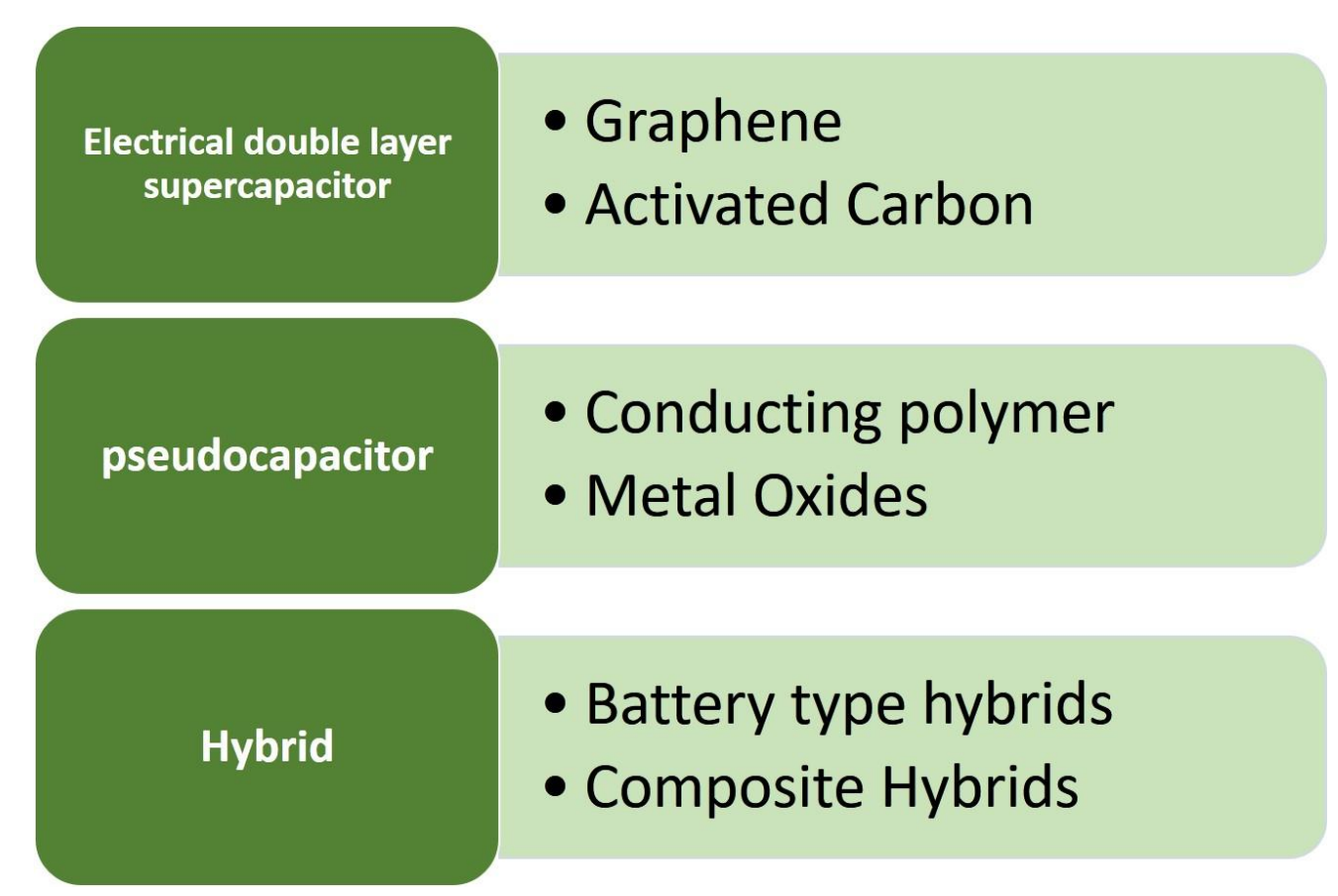

Figure 2. Classification of various types of supercapacitors.

\subsubsection{Electrical double layer capacitor (EDLC)}

In this kind of supercapacitors, the charges are stored non faradically, like conventional capacitors. At the interface of the electrolyte and electrodes, the charge separation happens and capacity is generated in this way. The main mechanism of these supercapacitors is surface dissociation and ion adsorption of both electrolyte and crystal lattice defects [3]. By applying the voltage charges are stored on the surface of electrodes. Due to the attraction of the unlike charges, the ions that are in the electrolyte diffuse through the separator and move toward the electrodes. Then a double layer of charges produced. by increasing the surface area and making the distances of two electrodes lower, higher energy densities are achieved. This kind of supercapacitors as is shown in fig. 3, have high cyclic stability because there is no charge transfer between electrodes and electrolytes. So, there is no composition degradation and charge storage is reversible. Both aqueous and organic electrolytes can be used in these supercapacitors. The electrodes of these supercapacitors are usually made of high surface area and porous structures as carbon based materials [3]. 


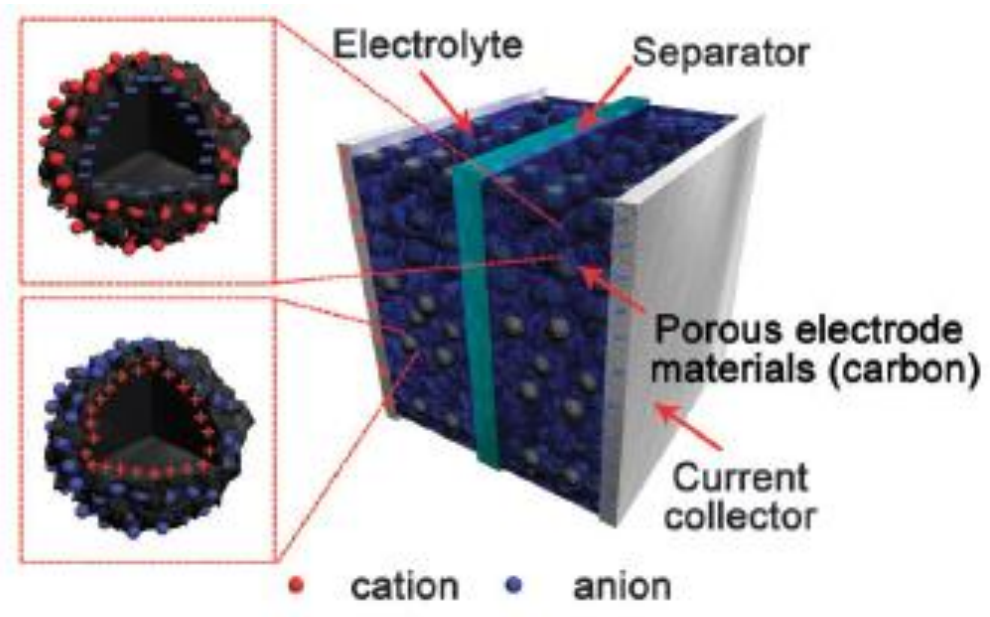

Electrical double-layer capacitor

Figure 3. Schematic of an electrical double layer capacitor [4].

\subsubsection{Pseudo supercapacitors}

The charge storage in these supercapacitors is based on a reversible faradic redox reaction that happens at the surface of the electrodes and charges are passing through the double layer. Therefore they can store more specific capacity. Conducting polymers or metal oxides such as $\mathrm{MnO}_{2}, \mathrm{RuO}_{2}$ is the type of the electrodes that are used in these kinds of supercapacitors [5]. Fig. 4 is the schematic of Pseudo supercapacitors. As another type of supercapacitors, pseudocapacitors have cones and pros. The advantages of these supercapacitors are their high capacitance because the electrochemical reactions happen both on the surface and the bulk near the surface. One of the important disadvantages of pseudocapacitors supercapacitors is mechanical changes in their electrodes like swelling or shrinkage which leads to poor cycle life [3]. 


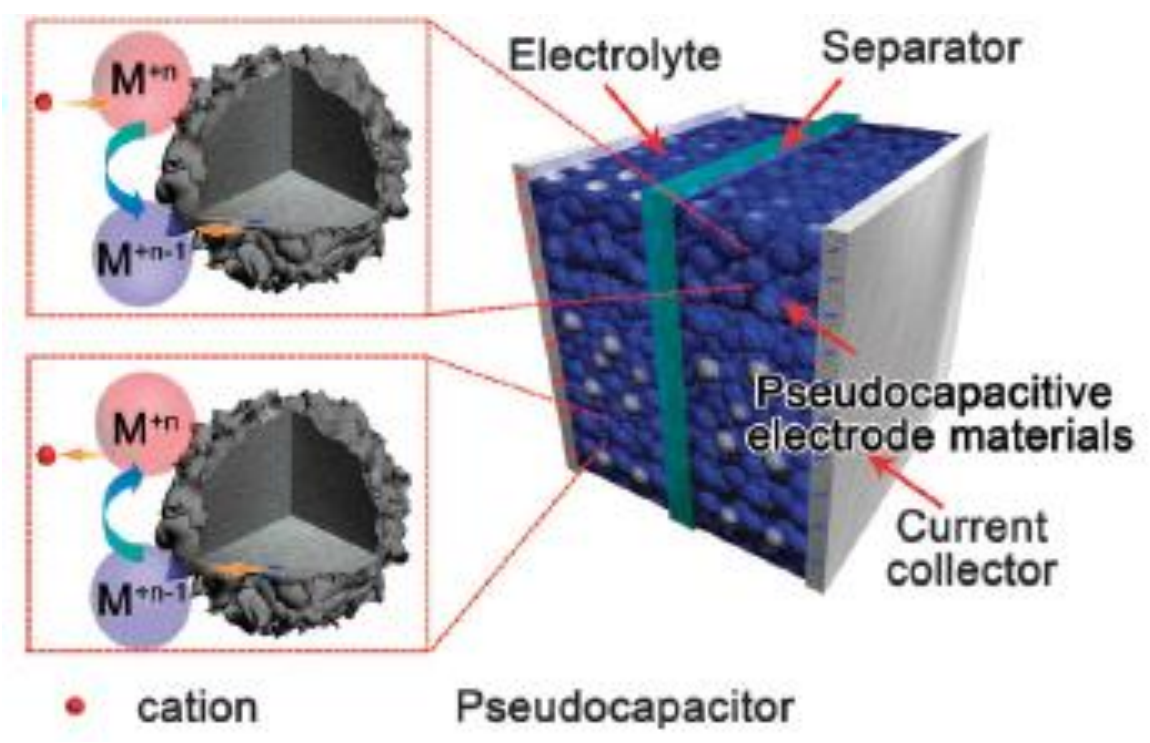

Figure 4. Schematic of a pseudo supercapacitor [4].

\subsubsection{Hybrid Supercapacitors}

As it is shown in fig. 5, this kind of supercapacitor is produced to increase the advantages and lessen the disadvantages of the two previous supercapacitors. In hybrid supercapacitors the charge storage system is based on both electrical double-layer and faradic mechanism. They can have better and higher energy and power densities than EDLC without having any problem in cycling stability as pseudocapacitors. Some new battery type materials are examples of this kind of supercapacitors [4].

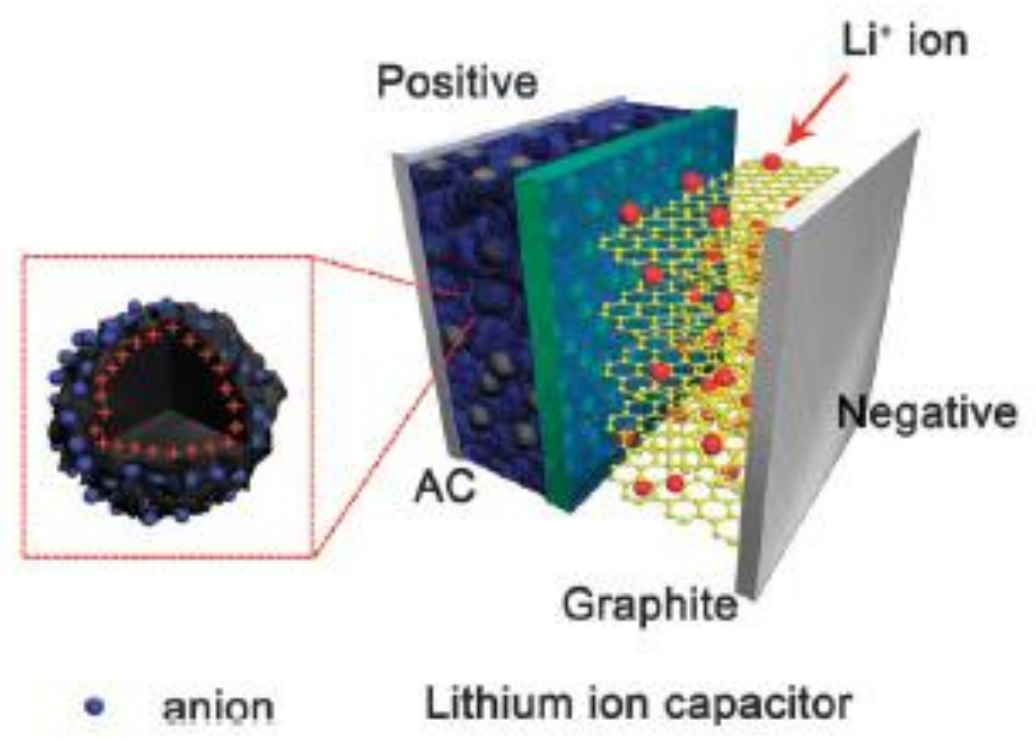

Figure 5. Schematic of an hybrid supercapacitor [4]. 


\subsection{Structure of the Supercapacitors}

In this thesis, the design of the supercapacitors are somehow similiar to battreies. It consists of the two electrodes, electrolyte and the seperator. The important part of the supercapacitors are the electrodes. The main properties of the supercapacitors are mainly based on the type of the electrodes that have been used. Fig. 6 is showing the schematic of a battery-like supercapacitor and fig. 7 is the schematic of the inside of supercapacitor which the particles are seperated inside the electrolyte.

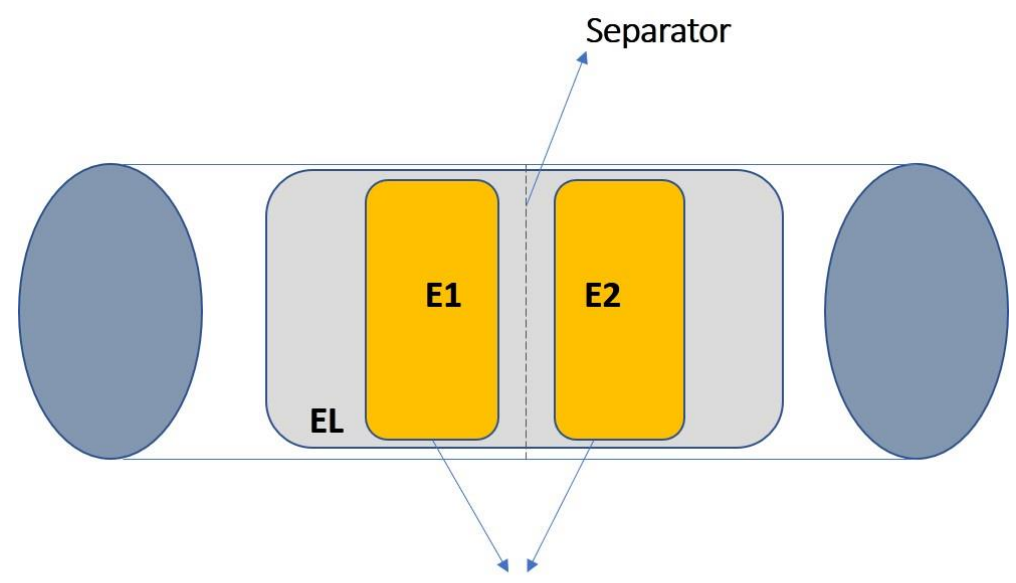

Electrodes

Figure 6. The general schematic of supercapaitor E1 and E2 are the electrodes and EL is the electrolyte.

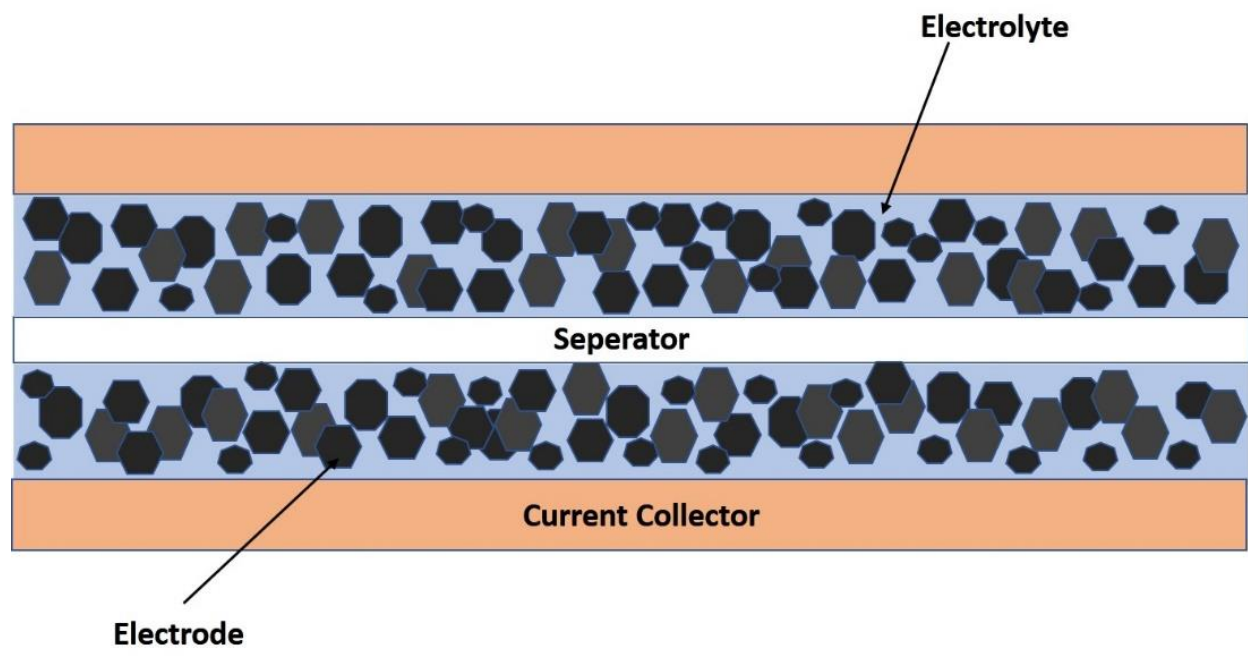

Figure 7. The Shematic of inside of the symmetric supercapacitor. 
Therefore, one of the aims of this work is to find a way to increase both the power and energy density of the supercapacitors by using the synergy of both 3D graphene foam (GF) and ball milled $\mathrm{ZnO}$ nanoparticles.

\subsubsection{Electrodes}

\subsubsection{Carbon based materials}

Graphite is a crystalline form of carbon in which the atoms are arranged naturally in hexagonal form [4]. Graphite possesses high thermal as well as electrical conductivity and that is the reason it is mostly employed as an electrode or in the applications of high temperature materials processing [6]. Graphene oxide (GO), on the other hand, is a two dimensional combination of $\mathrm{sp}^{2}$ bonded atom with an extended layered structure, which is produced from controlled oxidation of graphite. In its structure, GO has hydrophilic polar groups containing hydroxyl, carboxyl, aldehyde groups, and epoxy groups which provide some interesting features such as intercalating and swelling [7]. Additionally, due to its chemical stability, specific surface area, flexibility, and environmental friendly properties, GO has attracted great interest in different areas such as electrically conductive materials, supercapacitors and porous absorbents $[7,8]$. Reduced $\mathrm{GO}$ ( $\mathrm{rGO}$ ), however, is obtained by different methods of reducing the GO. The aim of reduction is to make the rGO structure and properties similar to pristine graphene [9]. There are three ways of reduction: thermal, electrochemistry and chemical, each of which has its own advantages and disadvantages. Thermal reduction, for example, requires very high temperature, whereas electrochemical reduction demands much more energy [7]. As a result of removing oxygen function, transparency and dispersibility of rGO is very low. Also, in the reduction process, polar functional groups of $G O$ is removed so its hydrophilic nature changes to hydrophobic, consequently rGO becomes hydrophobic [8]. In addition, the color of yellow-brown suspension of GO changes to black which can be a result of reduction in polar function of the GO's surface. The ClO ratio of GO is 4:12:1 depending on the preparation method. This ratio changes to 12:1 in rGO [9]. Impurities and defects, as an inevitable part of crystals, affect their characteristics such as electronical, mechanical, optical, and thermal properties. Graphene is not an 
exception and similar to many other materials contains several defects in its structure. These defects can have motions in parallel to the graphene plane, which can be low or high based on the type of the defect. One type of these defects is point defects, for which Stone-wales defect is one of the simplest example. In this defect, as the result of $90^{\circ}$ rotation of a C-C bond, four hexagon changes into two pentagons, the number of atoms would be the same as pristine graphene and no change in the number of the atoms would occur [10]. The other type of point defect in graphene is single vacancy in which graphene would miss one of its atoms, and due to this loss there should be two or three dangling bonds. Moreover, the formation energy of this defect is $E_{\mathrm{f}}=7.5 \mathrm{eV}$ which is a very high energy compare to other materials [10]. On the other hand, double vacancies occurs after removing two neighboring atoms, where two pentagon and one octagon appear and the formation energy would be close to the single vacancy. Although, double vacancies are stable in low temperatures, by increasing the temperature they will change to mobile defects. In contrast, carbon adatomic occurs by the interaction of carbon atoms with graphene layer, where two covalent bands will be formed between the surface atoms and atoms in the graphene plane [10]. In other words, the adatom has a bridge-like structure between two surface carbon atoms [11]. These defects can move on the surface of the graphene even at room temperature. Also, foreign adatoms happens when a noncarbon atom have some bonding with graphene. They can act as substitutional impurities so one or two carbon atoms are replaced by the impurities. Since Boron and Nitrogen have one electron less or more than Carbon, they can act as a natural dopants in graphene and can cause a change in its electronic structure. One dimensional defects like dislocation defects are the other type of defects seen in graphene. They separate domains of different crystal orientations. There are also some defects at the edge of the graphene, where there are two simple edge structures: armchair and zigzag. These two can decrease the dangling bonds to minimum and that's the reason these two orientations are preferred. Fig. 8 is the schematic of the defects in graphene. 


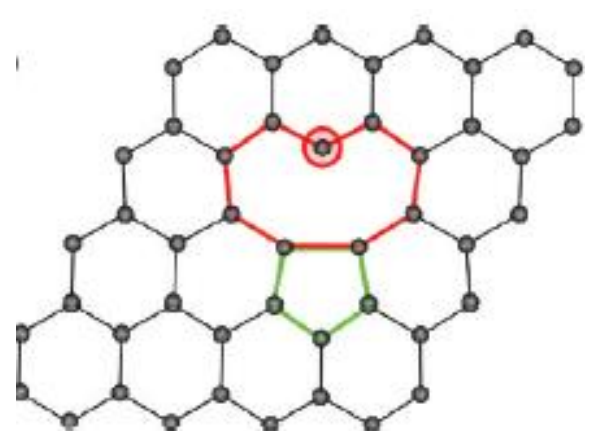

(a)

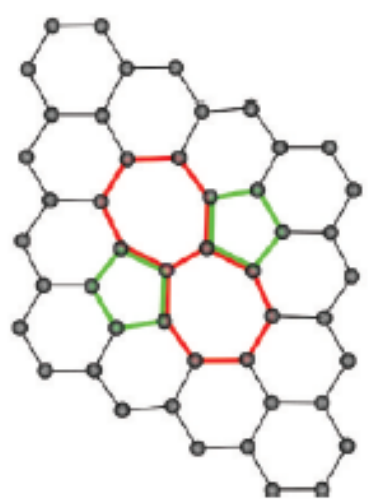

(c)

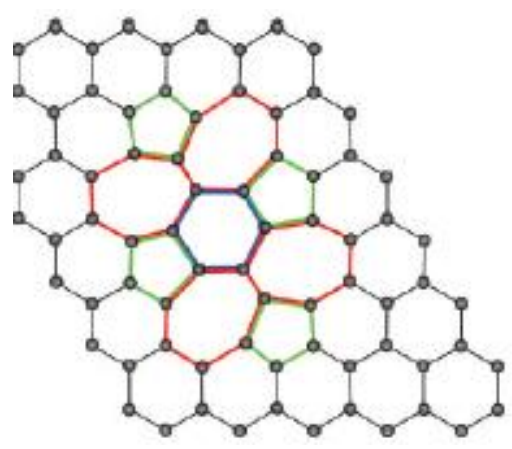

(b)

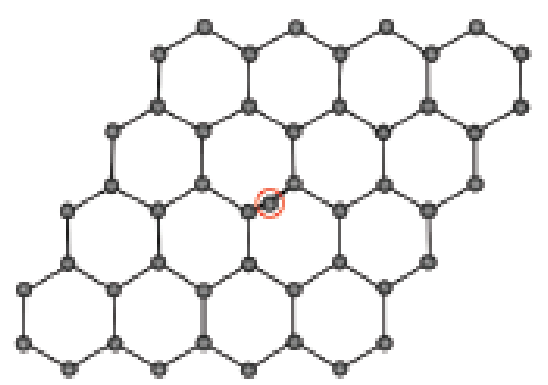

(d)

Figure 8. Different types of defects in Graphene: a) vacancy, b) divacancy, c) Stone-wale, d) adatom [12].

In order to comprehend the scattering of light as well as the emission and absorption of phonons in graphene, Raman spectroscopy is employed [12]. Among all the $\mathrm{sp}^{2}$ carbon materials, the simplest spectrum belongs to monolayer graphene. There are two features in the spectrum, G-band and 2D-band. A proper way to detect the defect density in graphene is using the ratio of the intensity of disorder-induced D-band to the symmetry-allowed G-band ( $\left(\mathrm{D} / \mathrm{l}_{\mathrm{G}}\right)$. In other words, by analyzing the D- and Gbands, we can gain information about crystal structure and physical properties of the graphene. In detail, by using laser excitation, the G-band generally appears around $1600 \mathrm{~cm}^{-1}$. This band is coming from first order Raman scattering and appears due to the double degenerate phonon mode at Brillouin zone. On the other hand, the 2D band appears around $2700 \mathrm{~cm}^{-1}$ and is produced due to the second order process 
[12]. Because of the disorderness in the structure of graphene, some vibrational modes would be activated. The D-band appears in the spectrum around $1400 \mathrm{~cm}^{-1}$ because of the defects or disorder [13]. Thus, in high crystallinity of graphene the D peak can be Raman inactive. However, even in defect-free samples, the D-peak may appear at the edges since the edges act like imperfections. Though, a perfect zigzag edge cannot cause D-peak due to the momentum conservation [14]. Raman spectroscopy is able to distinguish single layer graphene from multi-layer ones. Bilayer graphene, for example, has a wide 2D-band in comparison with monolayer graphene [15]. Fig. 9 is the raman spectroscopy of Graphene and graphite with the $G, D$ and 2D peaks.

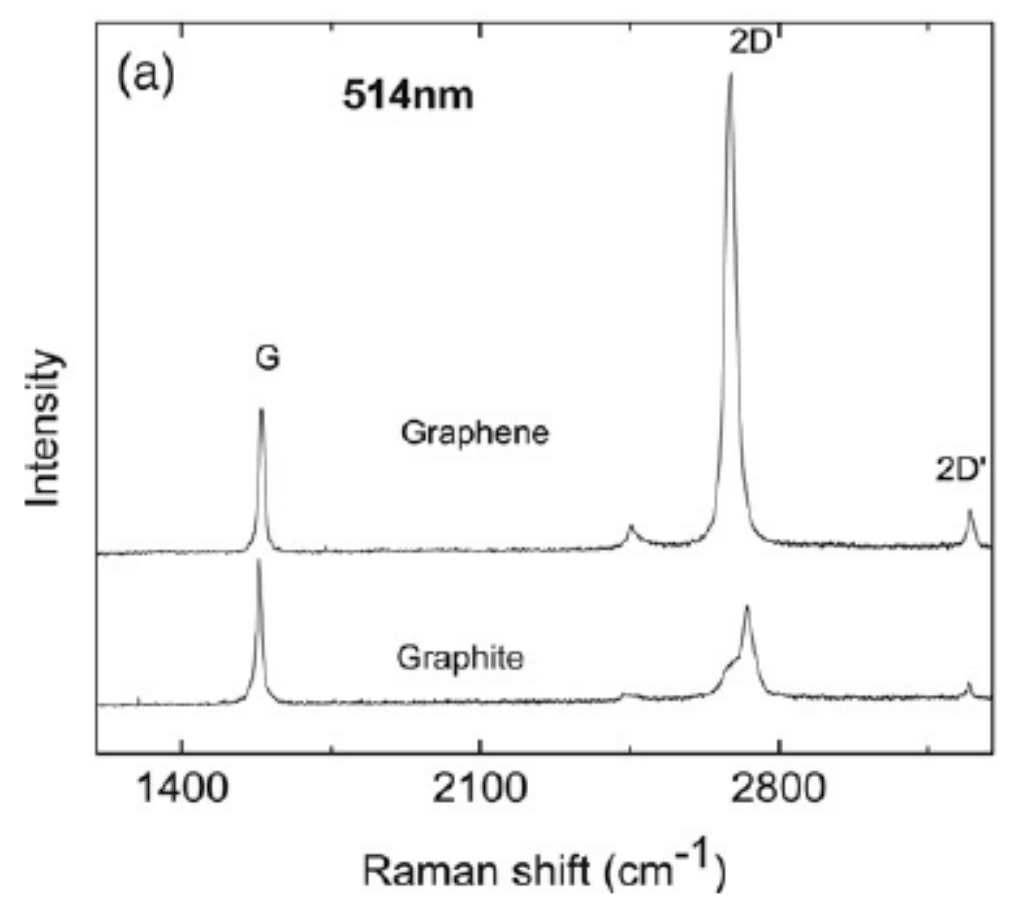

Figure 9. Raman spectroscopy of graphite and graphene [16].

In addition to graphite, $\mathrm{GO}$ and $\mathrm{rGO}$, one of the prominent carboceous material is called graphene foam (GF) which is one kind of 3 dimensional (3D) graphene based material that have been developed recently [17]. GF has the same properties as graphene as well as some other superior properties such as higher surface area, mechanical strength, electrical conductivity and interconnected porous structure . There are different ways for producing GF such as chemical vapor deposition CVD [17], electrostatic spray deposition [18], electrophoretic deposition [19], and ink-jet 
printing [20-22]. Among all mentioned methods, in present work, GFs synthesized via CVD technique on Ni foams which is an efficient way of process to synthesize the 3D foams.From the electrode performance point of view, graphene base materials show a high power density and good electrical conductivity and fast charge transfer [23, 24]. However, using these materials alone as an electrode can have some disadvantages. The important cone is their high internal resistance that cause due to the contact resistance of carbon particles [25, 26]. The other limitation is agglomeration of the graphene sheets due to the Wan der Waals attraction and as a result, reduction of surface area, so, some part of their surface area is unreachable to the electrolyte, that leads to low capacitance performance [2, 26, 27]. The solution of this problem is using metal oxides at least for the second electrode to prevent graphene sheets from restacking and agglomeration [24].

\subsubsection{Zinc Oxide}

$\mathrm{ZnO}$ is one of the most important transition metal oxides that attracts lots of attention to itself [28]. It has lots of application in different fields such as solar cells, gas sensors, capacitors, photoprinting, nanodevices and in cosmetic creams and sunscreen [29]. ZnO mostly known as II-VI semiconductor, as in the periodic table, $\mathrm{Zn}$ is classified in group two and oxygen is classified into group six. In addition, it is ntype semiconductor with three different crystalline form: hexagonal (wurtzite), regular (zinc blende), and rarely observed regular (rock-salt) as it is shown in fig. 10 [30].The most common structure is wurtzite structure which belongs to the spatial group of $\mathrm{C}_{4}$ with the lattice constants of $a=0.324 \mathrm{~nm}$ and $c=0.521 \mathrm{~nm}$ at room temperature [26]. At the corner of the tethrahedron, anions are surrounded by four cations. 


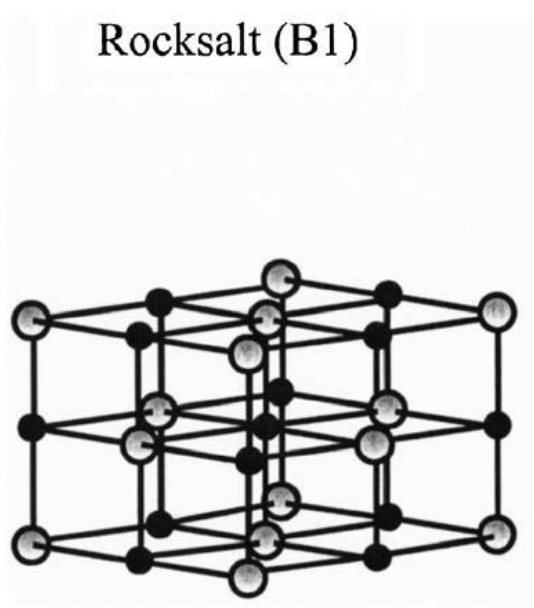

(a)

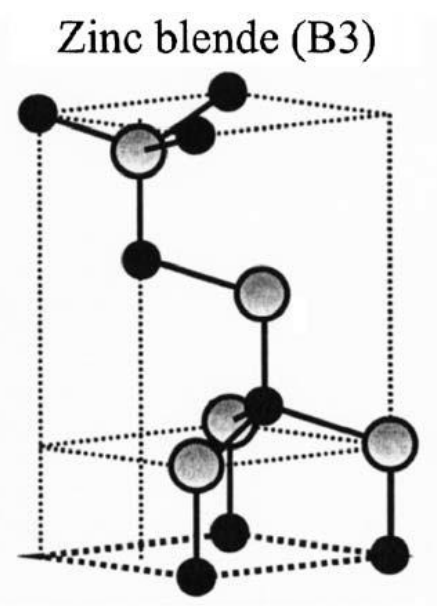

(b)
Wurtzite (B4)

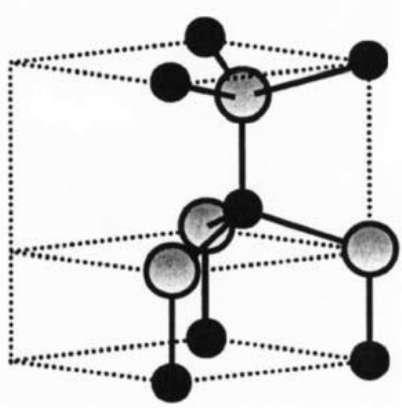

(c)

Figure 10. Different crystalline structures of $\mathrm{ZnO}$ [29].

The significant property of the $\mathrm{ZnO}$ is its wide and direct band gap energy $\left(E_{\mathrm{g}}=3.4\right.$ $\mathrm{eV}$ at $300 \mathrm{~K}$ ) which make it a potential candidate for electrodes in supercapacitors [20, 31-33]. Since the $\mathrm{ZnO}$ is highly defective material intrinsically the electrons or holes can be trapped in the bandgap which gives rise extra room for the charges to form higher capacitance. There are methods for synthesizing nanocrystal $\mathrm{ZnO}$ such as hydrothermal synthesis [30], high energy ball milling [31], microwave-assisted synthesis [32], hydrolysis/condensation [33], and sol-gel processing [34]. Among all these methods high energy ball milling (HEBM) is the most cost efficient and simple way to producing nanoscale particles. By HEBM new kinds of defects can be initiated in the grain structure of the $\mathrm{ZnO}$ nanoparticles [31].

\subsubsection{Defect structure of $\mathrm{ZnO}$ and Graphene}

The plausable intrinsic defects in $\mathrm{ZnO}$ can be given as follows: (i) zinc vacancies, (ii) zinc on interstitial sites, (iii) oxygen on interstitial sites, and (iv) oxygen vacancies [20, 31, 34-41]. For understanding more about intrinsic defects in $\mathrm{ZnO}$, electron paramagnetic resonance spectroscopy (EPR) and photoluminescence (PL) spectroscopy can be used. EPR, can detect unpaired electrons in an applied magnetic field and shows them as signals in the spectrum with different $g$-factors. The $g$-factor of surface defects in $\mathrm{ZnO}$ is around 2.004 and $g$-factor related to core defects is around $1.96[31,39,42]$. This finding has been already well established as a core-shell model for $\mathrm{ZnO}$ nanoparticles obtained via EPR [40] Localization of 
electrons is related to their crystal structures, thus, their intrinsically created point defects. Localized electrons and the local position of defects are also interrelated to each other. For instance, a conduction electron or a free electron can be trapped in a vacancy of a crystal and become localized. Whatever, the physical or chemical trigger for such trapping mechanism is the electrons either will localized or delocalized. This can be investigated by microwave power dependence of EPR signal which is originating from the spins of unpaired electrons either localized or delocalized ones. Due to their local positions each defect center hence, electron spin will feel different magnetic fields during the EPR experiment and finally, resonate at different $\mathrm{g}$-factor. In general, localized electrons are free like electrons giving EPR signal around $g=2$ (near or at the surface) and the localized ones feel the strong crystal field which causes shift from $g=2$ values which is 1.962 for $\mathrm{ZnO}$ core. Once again, from the electrode point of view, $\mathrm{ZnO}$ is a suitable candidate for supercapacitor applications because of its good electrochemical activity, high power density, good cycling stability, low cost as raw material, and environmental friendliness among the various metal oxide materials [43, 44]. However, $\mathrm{ZnO}$ electrodes have their own limitations as well. At high rates, $\mathrm{ZnO}$ electrodes may show slow electron mobility because of its relatively high resistivity [43]. Therefore, one of the aims of this work is finding a way to solve such obstacles and increase both the power and energy density of the supercapacitors by using the synergy of both GF and ball-milled $\mathrm{ZnO}$ nanoparticles.

The defect structure in carbonaceous and semiconductors are more or less known. However, when a device was produced by the synergetic effects of two materials, it is highly difficult to realize how the intrinsic defects of each material contribute to the performance of the entire system. EPR spectroscopy can give valuable information about the competing effects that exist in synergic materials which are characteristic defects of the individual constituents of the whole system. Of course, when such a defective system is used in a device as a functional material the function and the performance of the device itself can be influenced dramatically due to the existence of such structural point defects. Therefore, at the synthesis stage, one must have extensive control of defect concentrations and possibly defect kinds. Therefore, complementary characterization techniques should be used to suspect reliable defect models. In addition to EPR, Raman and PL spectroscopy are also giving extra information regarding the point defects. In PL spectroscopy two distinct signals can 
be discussed. One is arising at around $\sim 380 \mathrm{~nm}$ due to the electron-hole recombination process which is closely related to the crystal quality and the other one is arising in the visible light range giving maximum at the green with a broadband which is closely related to the defect centers [31]. PL can nicely complement EPR results in terms of defects however it is important to note that EPR only gives information about the paramagnetically active defects and whereas PL gives information about all possible kinds of defects. Representative EPR and PL graphics of $\mathrm{ZnO}$ nanoparticles are given in Fig. 11 ( $\mathrm{a}$ and $\mathrm{b}$ ) where surface and bulk defects give rise to a well-known core-shell model [36, 37, 45].
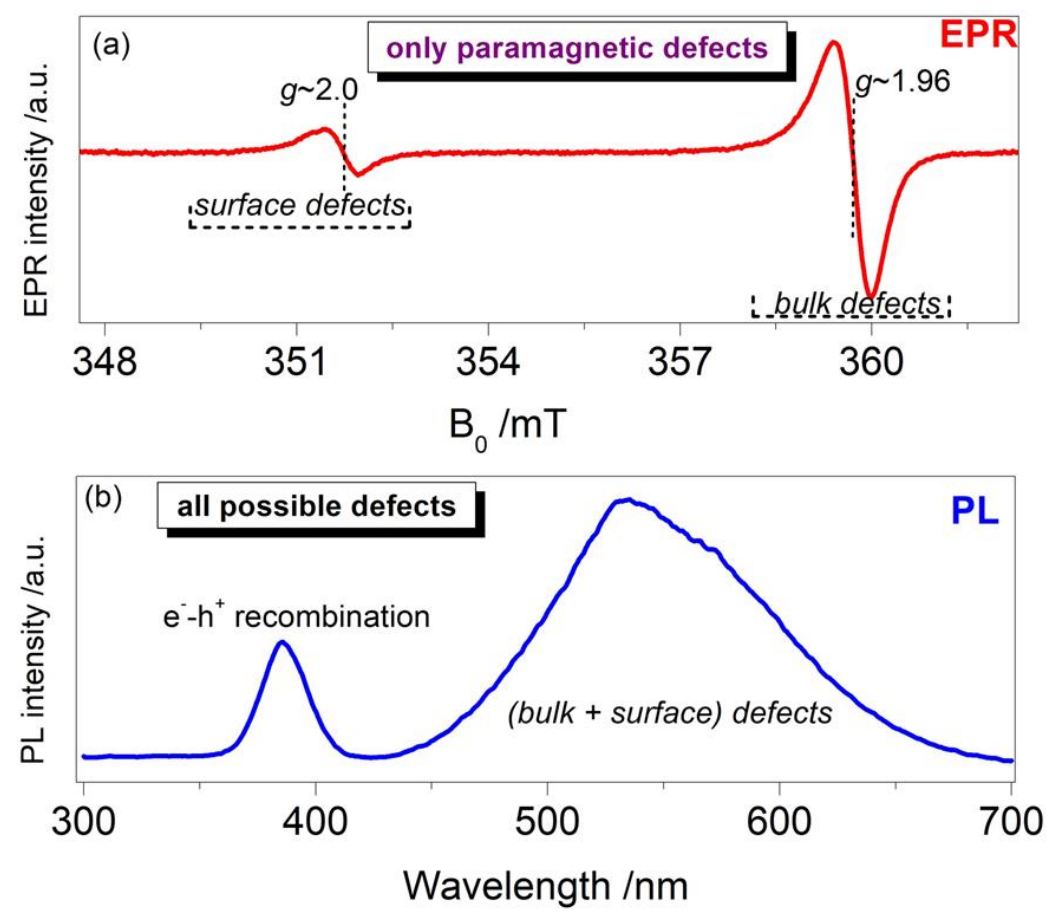

Figure 11. Representative EPR and PL spectra of defect structures in $\mathrm{ZnO}$ nanocrystal. In EPR only EPR active paramagnetic species can be observed whereas in PL all possible defect centers contributed to the defect-related visible emission band which is quite broad $(420 \mathrm{~nm}-700 \mathrm{~nm})$ [31]. 
One of the aims of this thesis is finding a way to solve obstacles related to carbon based and metal oxide electrodes and increase both the power and energy density of the supercapacitors by using the synergy of both GF and ball milled $\mathrm{ZnO}$ nanoparticles. In Chapter 2, the process of the synthesis of $\mathrm{ZnO}$ particles and GF as well as the design of supercapacitor is explained completely. In addition, the characterization method that is used during the whole experiment is described briefly. Chapter 3 is the explanation of all the results that are achieved in the experiment. For structural analysis, $x$-ray diffraction (XRD) and scanning electron microscopy (SEM) and, Raman spectroscopy is used. UV-VIS, electron paramagnetic resonance (EPR) and, Photoluminescence are used for electronic analysis. Finally, cyclovoltammetry and impedance for electrochemical analysis are done. The discussion parts are interpretation of all the characterization tests. Finally, Chapter 4 is a conclusion that achieved by the whole experiment and results. 


\section{Chapter 2}

2 . Experimental and Methods 


\subsection{Synthesis of $\mathrm{ZnO}$}

High energy ball milling (HEBM) is one of facile, cleanand the fast way of bringing the solid crystals into nanosize. This method is used for synthesizing semiconductors such as $\mathrm{ZnO}$, magnetic nanomaterials, carbon nanotubes and etc [29]. Basically, It is a way to attainng metastable materials and making particles better. For producing oxides of metal, ball milling is a good option since chemical reactions that normally dont happpen at room temprature can happen in ball milling tecnique [46]. the advantage of ball milling process over other synthesiz methodes such as thermal evaoration, vapor phase transport, dip coating is that growth tempratre of the materials are too low and the amount of the final product is high [47]. Moreover, it is a safe method without any gases emission through the process [29]. In addition, Starting from the bulk material one way to reach homogenously distributed crystal sizes down to $100 \mathrm{~nm}$. Normally, the performnce of the ball milling process is as follows: the powder get stuck between the highly kinetic balls and interior surface of the jars, as a result, grinding, deformation of the powder achieved [47]. Fig. 12 is the schematic of ball milling process. Sometimes, due to the long ball milling time, agglomeration happen and the particles size increases. The agglomeration is happening because of high surface area of the particles [48]. Many different factors can be effective in high energy ball milling for controling the mechanism of size: i) milling time ii) ball-to- powder ratio and iii) rpm iv) milling atomesphere v) temprature effect [48].

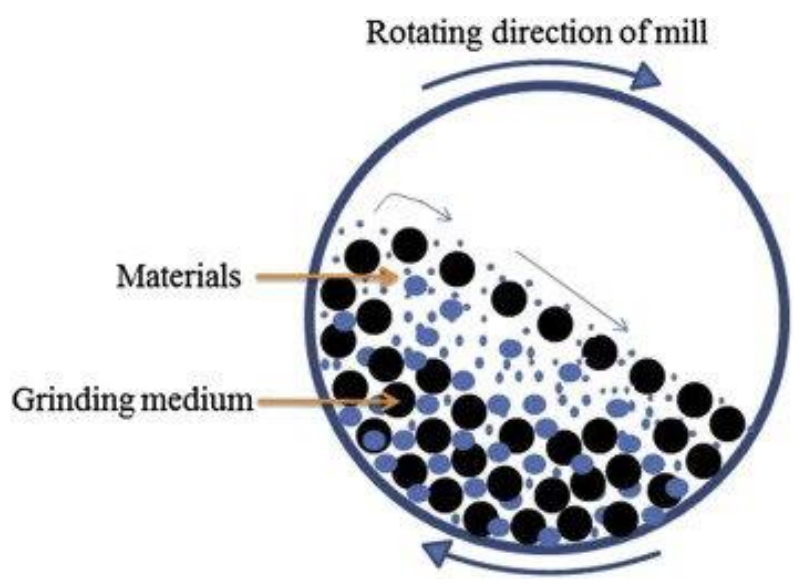

Figure 12. Schematic of ball milling powder [49]. 
In our study we keep the last two parameters fixed and change the milling time for controlling the crystal mean size. As a result, the longer the milling time the smaller the crystal size is. High energy ball milling method also enables to create defect structures because of high strain and to breaking of symmetry due to collision. Such collision of particles may also increase the temperature in the container and may cause agglomeration during the growth of nanoparticles. Overall, commercial (Merck, Germany, grade $99,99 \%$ ) high grade $\mathrm{ZnO}$ powder was used as starting material. The commercial powder was milled in a planetary-ball mill for various time periods in intervals $15 \mathrm{~min}, 30 \mathrm{~min}, 1 \mathrm{~h}, 5 \mathrm{~h}$ and $10 \mathrm{~h}$ with the speed of $2100 \mathrm{rpm}$ under atmospheric pressure at room temperature. Seven stainless-steel balls with diameter of $7 \mathrm{~mm}$ and 16 balls with diameter of $5 \mathrm{~mm}$ were used. The ball to powder ratio is $6: 1$ by weight. The ball milling procedure helped us to reduce the crystallite size of $\mathrm{ZnO}$ meaning that the longer the time the smaller the mean crystallite size. By this we obtained systematic decrease in particle size.

\subsection{Synthesis of 3D Graphene Foam}

3D-GF macrostructure was synthesized on a nickel foam with $1.6 \mathrm{~mm}$ thickness ( $\geq$ $95 \%$ porosity, $99.99 \%$ purity) via chemical vapor deposition (CVD) route. The nickel foam $\left(5 \times 5 \mathrm{~cm}^{2}\right)$ was placed horizontally in the central zone of the quartz tube in the CVD chamber and heated up to $1000^{\circ} \mathrm{C}$ with a flow of $\operatorname{Ar}(250 \mathrm{sccm})$ and $\mathrm{H}_{2}(100$ $\mathrm{sccm}$ ) gas mixture at low pressure (60 mbar). To remove oxide layer on its surface, the nickel foam was maintained for 10 minutes under such condition. Then, $100 \mathrm{sccm}$ methane $\left(\mathrm{CH}_{4}\right)$ gas as a carbon precursor was delivered into the quartz tube for $1 \mathrm{~h}$ to grow graphene sheets on the nickel template. Following this step, the sample was allowed to cool to ambient temperature naturally under Ar gas flow. In order to obtain the free-standing graphene macrostructure, the nickel template was etched in $1 \mathrm{M}$ $\mathrm{FeCl}_{3}$ solution for 1 day. After the etching, the 3D-GF was immersed in a distilled water- $\mathrm{HCl}$ mixed solution $(1: 1(\mathrm{v} / \mathrm{v}))$ to clean it from Fe residues. Finally, the GF was dried at $80^{\circ} \mathrm{C}$ for $30 \mathrm{~min}$. in an oven.

\subsection{Design of supercapacitor}

$\mathrm{ZnO}$ and 3D-GF materials were used as electrodes without any additives such as binders or carbon materials. This means there is no slurry phase in present capacitor design. Depending on whether the supercapacitor was symmetric or asymmetric, the 
combination of the electrode materials was changed. In order to exhibit all designs, Table 1 presents detailed information on the combinations of electrode materials. WHA1825042-Whatman ${ }^{\circledR}$ glass microfiber filters, Grade GF/F, were used as the dielectric separator; these filters are highly porous and effective to separate the two electrodes. Finally, well-known $1 \mathrm{M} \mathrm{LiPF}_{6}$ in a 1:1 mixture of ethylene carbonate and diethyl carbonate (Aldrich) electrolyte were used in order to get wide range of voltage window. The full cell supercapacitors with $\mathrm{LiPF}_{6}$ as the electrolyte were assembled in a glove box filled with $\operatorname{Ar}\left(\left[\mathrm{O}_{2}\right]<0.5 \mathrm{ppm}\right)$. The full cell design is illustrated in Fig. 13. These designs were successfully implemented in former studies based on octacalcium phosphate [50-53]

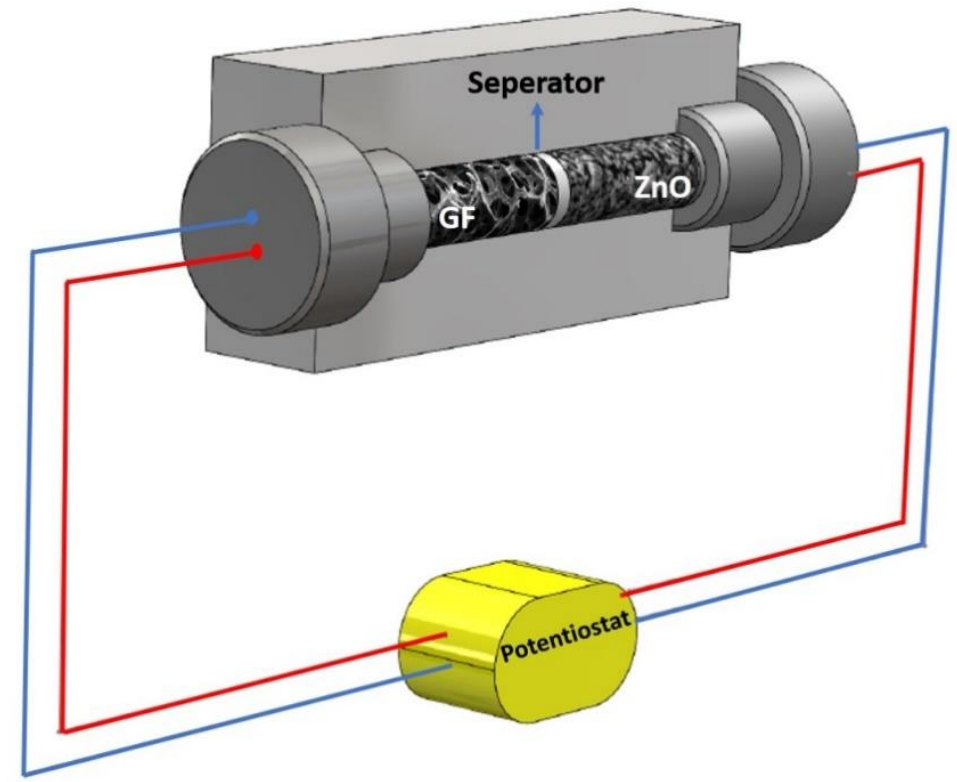

Figure 13. Graphical illustration of asymmetric supercapacitor model. The synergy of nanosized metal oxide $\mathrm{ZnO}$ and 3D-GF meets with the standard 1.0 M LiPF6 in $\mathrm{EC} / \mathrm{DEC}=50 / 50$ electrolyte deparated with glass fiber.

According to the design template given in Fig. 13 following supercapacitor devices were produced and tested by potentiostat and impedance (see Table 1). 
Table 1. Components of the various supercapacitors which are designed based on Fig. 13. Note that; LiPF6:1M lithium hexafluorophosphate dissolved in EC/DMC=50/50 (v/v).

\begin{tabular}{|c|c|c|c|c|}
\hline $\begin{array}{c}\text { Supercapacitor device } \\
\text { number }\end{array}$ & Electrode 1 & $\begin{array}{c}\text { Electrode } \\
2\end{array}$ & $\begin{array}{l}\text { Electrolyte } \\
\text { (EL) }\end{array}$ & Separator \\
\hline SC1 & bulk ZnO & 3D-GF & $\mathrm{LiPF}_{6}$ & $\begin{array}{l}\text { Glass } \\
\text { fiber }\end{array}$ \\
\hline SC2 & $\begin{array}{c}1 \mathrm{~h} \mathrm{HEBM} \\
\mathrm{ZnO}\end{array}$ & 3D-GF & $\mathrm{LiPF}_{6}$ & $\begin{array}{l}\text { Glass } \\
\text { fiber }\end{array}$ \\
\hline SC3 & $\begin{array}{c}10 \mathrm{~h} \mathrm{HEBM} \\
\mathrm{ZnO}\end{array}$ & 3D-GF & $\mathrm{LiPF}_{6}$ & $\begin{array}{l}\text { Glass } \\
\text { fiber }\end{array}$ \\
\hline
\end{tabular}

\subsection{Characterization Methods}

The crystalline structure and composition of materials can be obtained by X-ray diffraction (XRD). Since each material has its own diffraction pattern, XRD patterns known as fingerprint. Also, qualitive identification of phases, quantitative analysis of the phases, the size and shape of the unit cell are the other applications of XRD. Diffraction happens when destructive and constructive waves that scattered from a material interface each other. The diffraction from a crystal is described the Bragg equation:

$$
\mathrm{n} \lambda=2 d \sin \theta
$$

where $\lambda$ is wavelength and $\theta$ is the $x$-ray incidence angle, $d$ the spacing of the crystal planes and $n$ the diffraction order. The mean size of particles can be achieved by Scherrer formula by XRD too.

$$
d_{\mathrm{m}}=\kappa \lambda /(\Gamma \cos \theta)
$$

which $d \mathrm{~m}$ is the mean particle diameter, $\lambda$ the x-ray wavelength, $\Gamma$ the width (FWHM) of a diffraction peak, $\theta$ the diffraction angle, and $\kappa$ the Scherrer's constant being near unity for common crystals. In this work, X-ray diffraction (XRD) patterns were obtained by using Bruker, D2 Phaser with Cu-Ka radiation with a wavelength of 
$0.15404 \mathrm{~nm}$, voltage and current values of $40 \mathrm{kV}$ and $40 \mathrm{~mA}$, respectively. The data was collected at a range of $2 \theta=10-90^{\circ}$ and a step size of $0.02^{\circ}$.

Raman spectroscopy is a non-destructive method for studying vibrational properties of materials [54]. The whole process is based on the inelastic scattering. an incident light excited molecule in a material and the molecules can reflect light in different wavelength. Which means those scattered light that have lower wavelength than the wavelength of the source light is collected and is shown as Raman spectrum. Each peak is of the spectrum, is related to the special bond vibration of molecules [55]. In this work, Raman measurements were performed with a high-resolution inVia Raman Microscope (Renishaw inVia, excited by a $532 \mathrm{~nm}$ ).

The images in SEM as shown in fig. 14, can be taken by three ways: i) secondary electrons, ii) back scattered electron and iii) characteristic X-rays that enter in the detectors in the vacuum chamber. The source of the electrons is an electron gun that accelerated them and the electromagnetic lenses that can focus the electron. For focusing the electron beam on the surface of a sample, a scanning coil is used inside a chamber. When electron beam attacts the surface of sample, it interacts with the electrons of the surface and inside of the sample. The backscattered electrons (BSE) when collected at the detector, its signals are used to understand the distribution of different elements in the sample. The secondary electrons images are taken based on knocking the outer electrons of surface atoms on the sample surface and all collected at the SE detector. On the other hand, the characteristic x-ray is another type of emission that occurs because of the interaction between the accelerated electron and the electrons that are in the inner shell of the atoms. The $x$ ray emission is happening due to the removing and replacing of electron with higher energy electrons. The composition of different elements in the materials can be recognized by taking with this method [56]. Here, the morphology of GF and ZnO particles were observed by the aid of Scanning Electron Microscopy (SEM) (JEOL, JSM-6010LV) at $5 \mathrm{kV}$. 


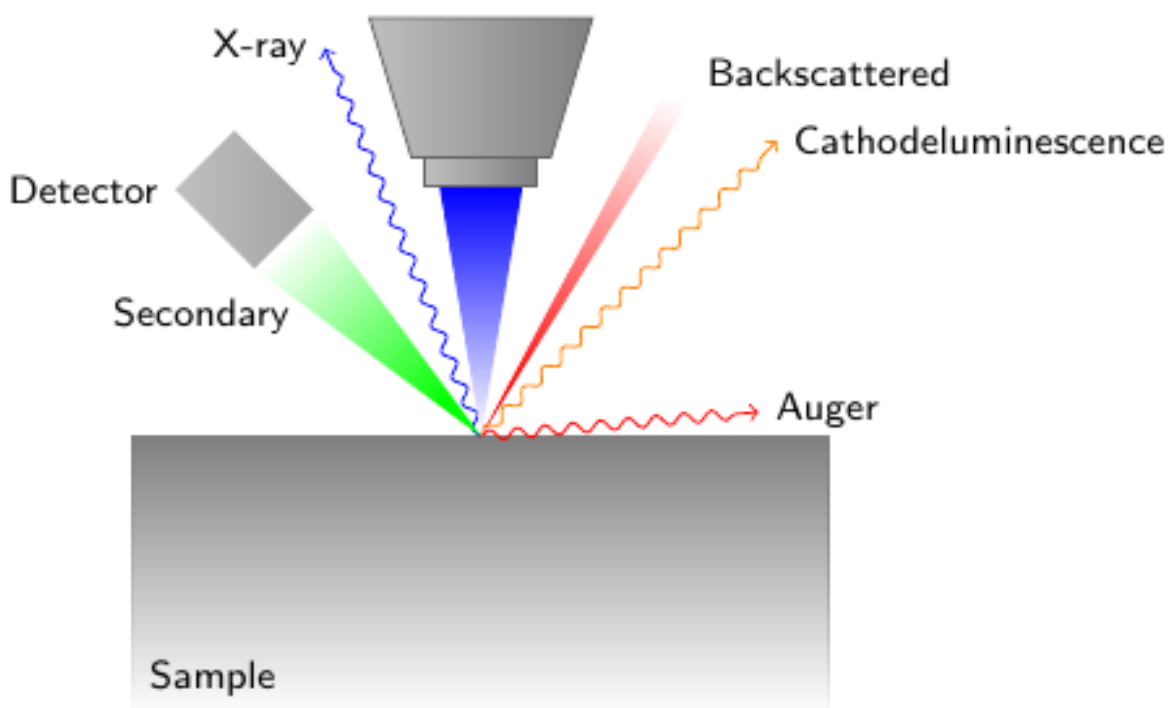

Figure 14. Incidence of electron beam on the sample and creation of secondary electrons, back scattered electron and characteristic X-rays [56].

The study of upaired electrons in materials can be achieved by electron paramagnetic resonance spectroscopy (EPR spectroscopy) or electron spin resonance spectroscopy. The basic concepts of the technique is the electron spins that are excited instead of the spins of atomic nuclei and is very practical method for studying metal complexes or organic radicals and for paramagnetic compounds with an odd number of unpaired electrons. EPR spectroscopy can be used when there are an even number of unpaired electrons [57].

EPR spectra of samples are described by a phenomenological spin Hamiltonian:

$$
\hat{H}=\beta \hat{\overrightarrow{\vec{S}}} \mathrm{~g} \vec{H}+\hat{\vec{S}} \mathrm{~A} \hat{\vec{I}} \ldots
$$

where $\beta e$ is the electron Bohr magneton, $S^{\wedge}$ and $I^{\wedge}$ are the electron and nuclear spin operators, respectively, and $g$ and $A$ are $3 \times 3$ matrices that define the anisotropic (orientation-dependent) interaction of the unpaired electron with the external magnetic field, B, and with nuclear spins, respectively. The $g$-tensor simplifies to a scalar quantity of 2.0023 for a free electron [58].The process of this device is as following: a sample is irradiated with microwave radiation, and the field is swept until resonance occurs. A a result, some electrons that are in lower energy level excited to the upper energy level. There should be an external magnetic field for occuring the 
transition. So, the sample is exposing to a fixed frequency of microwave irradiation and an external magnetic field.a resonance condition happens when the magnetic field and the microwave frequency are "just right" to produce an EPR resonance (or absorption) [59].The field at which resonance occurs depends on the number of unpaired electrons, the geometry about the metal center, and the metal ligands [57]. In this work, EPR measurements were performed with a Bruker Benchtop EMX Nano EPR spectrometer with an integrated referencing for $g$-factor calculation and also integrated spin counting unit. The microwave frequency of the resonator was 9.41 $\mathrm{GHz}$ and all spectra were measured with $5 \mathrm{~mW}$ microwave power at room temperature.

Electrical impedance spectroscopy (EIS) measurements is one of the important methods in electrochemical researches. The importance of this technique is that it can divided the effect of various materials that have contribution of double layer, electron transfer resistance. Basically, Electrochemical impedance is the response of an electrochemical system (cell) to an applied potential. the measurement is obtained by applying $A C$ voltage to the system and then measuring the current through the system. in EIS measurements, both $V$ and $I$ are sinusoidal functions which means that they are time dependent. A single frequency potential input with amplitude, $V_{o}$, and radial frequency, $\omega$, can be expressed as:

$$
V(t)=V_{0} \cos (\omega t)
$$

where $V_{t}$ is the potential at time $t$ [60]. In this work, EIS measurements were carried out using a Versastat IV potentiostat (Princeton Applied Research) in atmospheric pressure at room temperature in the frequency range between $0.1 \mathrm{~Hz}$ and $100 \mathrm{kHz}$. For analyzing the spectra we used the Versa Studio V2.4 software. The amplitude of voltage modulation was set to $100 \mathrm{mV}$. In the present case, a four-point electrodesetup was used.

UV-vis is absorption or reflectance spectroscopy in the region of ultraviolet visible. In this region of the electromagnetic spectrum, atoms and molecules sustain electronic transitions. The absorption or reflectance can be determined by their colors in the visible range. Fluorescence spectroscopy encounters with transitions from the excited state to the ground state and absorption measures transitions from the ground state to the excited state. The process of the device is as following: light 
incident with the material then a part of the light is absorbed inside the molecules and as a result, electrons excite to the higher energy level. degree of absorption by a material is recorded by the spectrometer at different wavelengths. Finally, the spectrum is plotted as absorbance $(A)$ versus wavelength $(\lambda)$. The maximum amount of light that absorbs at specific wavelength is known as $\lambda$ max [61].

Photoluminescence spectroscopy (PL) is a method for understanding electrical structure of materials. The advantage of this method is its non- contact and nondestructive way of probing. Light is emitted to the sample and it is absorbed by the sample. As a result, some electrons are excited and move to the to the other higher energy states. The emission of light that is released due to the returning of the electrons to their equilibrium, lower states is called photoluminescence. The amount of released energy is related to the energy difference of excited and equilibrium state. Fluorescence and phosphorescent are two type of photoluminescence. The differences are the emission time. The emission in the fluorescence is immediately while the emission in phosphorescent took longer [62]. This method is very important, especially in semiconductors, because the purity of the material can be detected in this way [63]. 


\section{Chapter 3}

\section{Results and Discussions}




\subsection{Structural and electronic analysis}

\subsubsection{XRD Characterization}

One of the best ways to perform structural analysis on nanostructured systems is to apply XRD. Once chemical synthesis is done XRD is crucial for understanding the symmetry, purity, size and strain effects. Fig. 15 shows the XRD pattern of both 3DGF and $\mathrm{ZnO}$ crystals before $(0.5 \mathrm{~mm})$ and after HEBM. For GF sample two diffraction peaks located at $\sim 26^{\circ}$ and $\sim 54^{\circ}$, namely (002) and (004) represent graphitic carbon. The results of $\mathrm{ZnO}$ powders show that all the powders are purely $\mathrm{ZnO}$, and no contamination such as metal ions or foreign metal oxide phases occur during the ball milling process. This is because all the $\mathrm{ZrO}_{2}$ balls that were used during ball milling are ultrasonicated and cleaned carefully before using. Moreover, all the peaks in the XRD pattern of samples correspond to the Wurtzite structure of $\mathrm{ZnO}$ of hexagonal phase and P6 ${ }_{3} m c$ point-group symmetry (JCPDS file or ICDDPDF code: 36-1451). The average crystallite size of the $\mathrm{ZnO}$ was determined from the X-ray peak (101) broadening using a Voigt peak profile analysis after eliminating the instrumental broadening and strain contribution. Such determination of size is based on the wellknown Scherrer equation[64]. Fig. 15b depicts the decrease in crystallite size with increasing milling time. Increasing the milling time beyond $600 \mathrm{~min}(10 \mathrm{~h})$ did not lead to any further significant changes in the XRD feature. It is also here very important to note that with a very little mechanical energy one may reach from $500 \mathrm{~nm}$ to $50 \mathrm{~nm}$ in a couple of minutes by just HEBM procedure. This makes the technique quite favorable and facile for a top-down approach of nanoscale synthesis. 

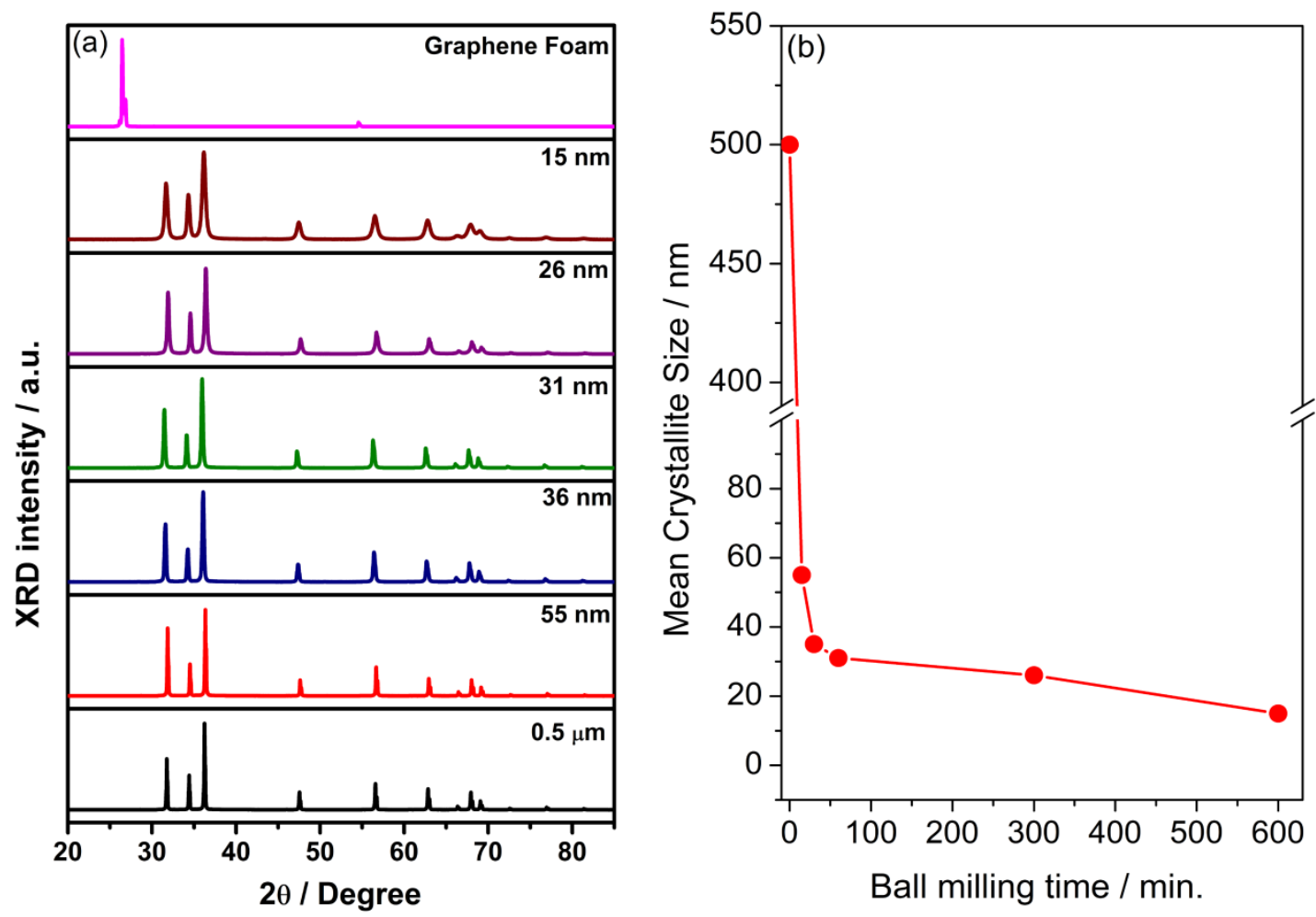

Figure 15. a) XRD patterns of 3D-GF and HEBM ZnO nanocrystals. Different milling times result in different average sizes. b) HEBM holding time with respect to mean crystallite sizes obtained from XRD profiles. The figure revealed that with a very low mechanical e energy it is possible to reach from micron size down to a few tens of nm size.

\subsubsection{SEM Characterization}

As it is obvious from Fig. 16a, the porous 3D skeleton was obtained via the CVD method and the nickel foam as substrate was removed completely. Also, SEM images of $\mathrm{ZnO}$ particles at different magnifications are shown in fig. 17. By increasing ball-milling time the particle size decreases. As shown in the pictures, milling effect on the size and shape of the particles is more significant after $1 \mathrm{~h}$ of ball milling. 

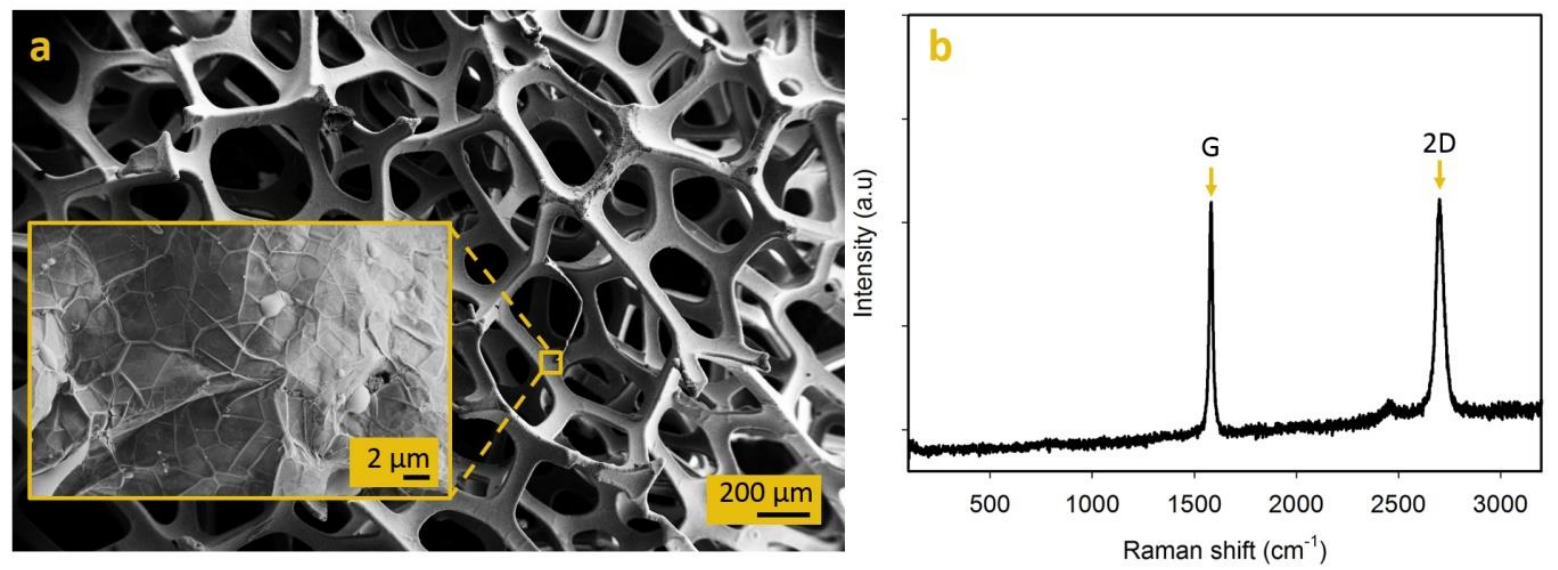

Figure 16. (a) SE-SEM images of 3D-GF with two different magnifications indicating porous structure. (b) Raman spectra of 3D-GF indicating $G$ and 2D bands. The defect-related D band is absent. 


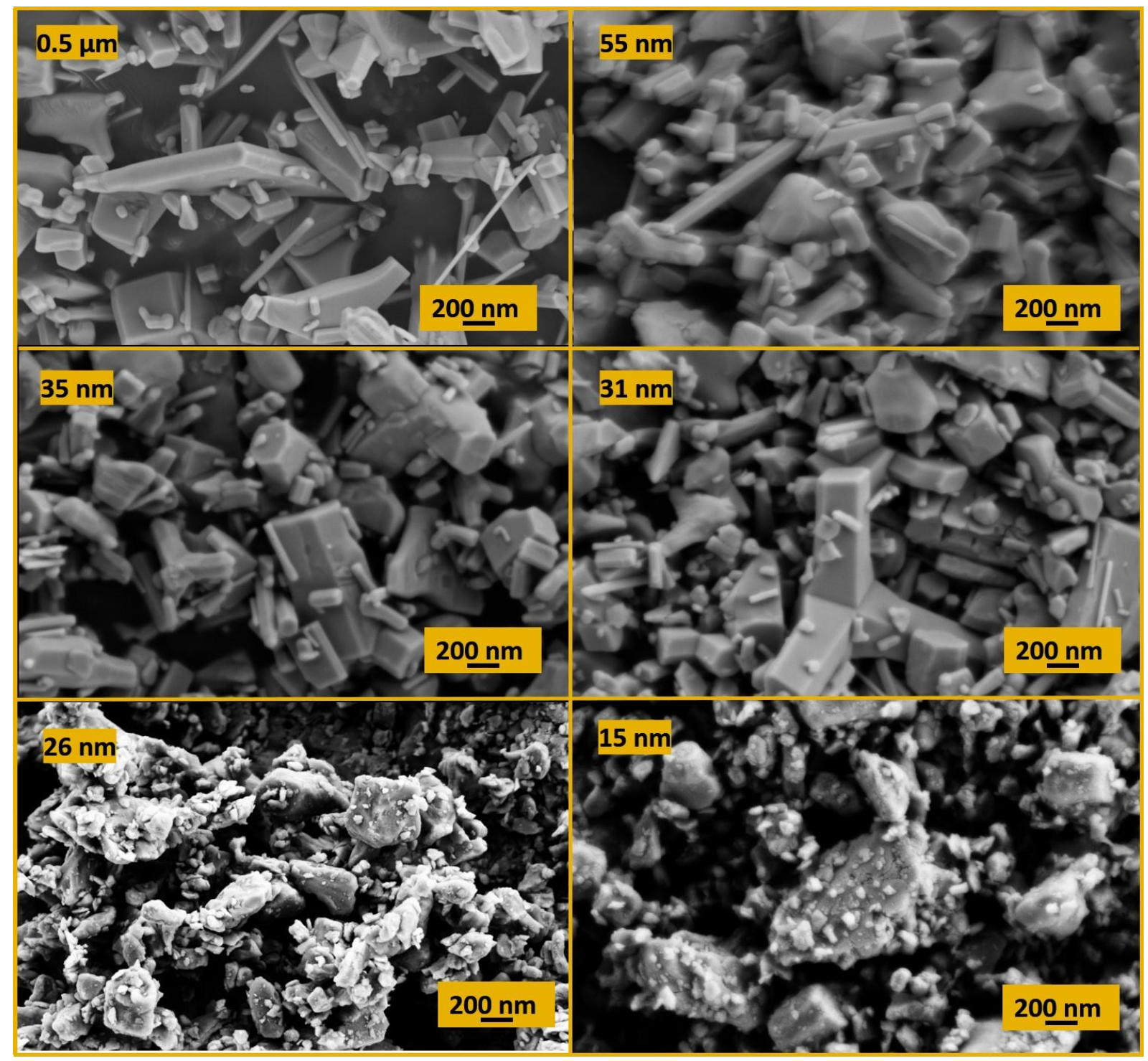

Figure 17. SEM images of HEBM ZnO nanocrystals indicating hexagonal structure. By decreasing average size to sub-30 nm, strong distortions occur from the hexagonal symmetry.

\subsubsection{EPR and PL Characterization}

EPR spectroscopy is one of the most powerful methods to detect the point defects directly in solid-state samples. In principle, $\mathrm{ZnO}$ has paramagnetically active defect centers namely "singly or doubly ionized Vo", "zinc vacancies $\mathrm{Vzn}$ ", and "interstitials of $\mathrm{Zn}$ and $\mathrm{O}\left(\mathrm{Zn}_{\mathrm{i}}\right.$ and $\left.\mathrm{O}_{\mathrm{i}}\right)$ ". The EPR spectrum of bulk $\mathrm{ZnO}$ gives two distinct EPR centers at $g=1.962$ and $g=2.006$ depending on their localization in the $\mathrm{ZnO}$ nanocrystal lattice. The ionized electrons or holes which are near the surface are mostly delocalized and act like free-electrons and their $g$-factor is around 2.006 ( $g$ treeelectron=2.0023). Meaning that the effect of orbital angular momentum contribution to 
the total angular momentum is too less. On the other hand, the electrons which are in the center of the crystal, they are more bound and feel larger spin-orbit coupling effect. That's why the $g$-factor has a large deviation from 2 to 1.962 [20]. Fig. 18a shows the EPR spectra of both electrode materials that are used in supercapacitor devices that are designed here. At first, 3D-GF has no significant EPR signal which means that the defects are either too less and remained under the detection limit of EPR or the GF samples are highly uniform and defect-free. Indeed, the latter is true while the Raman spectrum in Fig. 16b also does not reveal any defect related to Dband. Since EPR and Raman have different detection sensitivity and different transition rules one can easily exclude the assumption of low concentration of defects. Indeed, these two techniques complement each other and satisfy that GF that are used for electrode here are completely defect-free. On the other hand, Fig. $12 \mathrm{a}$ shows the evolution of defect structures in ZnO obtained via EPR spectroscopy. We see two distinct EPR spectra at $g \sim 2$ and $g \sim 1.96$ and by increasing the milling time, hence decreasing the mean particle size the defect centers on the core gradually decreases and the defect centers on the surface increases. This is naturally expected effect according to a well-known core-shell model. To see the effect in a more clear way we considered in Fig. $18 \mathrm{~b}$ the 55 and $15 \mathrm{~nm}$ size $\mathrm{ZnO}$ nanocrystals and integrated their first derivative signal and obtained absorption EPR signal. The absorption signal indicates the strong anisotropy in the signal indicating three different kinds of defect signals at the surface having $g$-factors of 2.0045, 2.0130, 2.0182. These $g$-factors are quite close to each other and mean that ball milling causes high strain and due to this high strain at the nanoscale, the defects can take place several different localization thus different site symmetries. Such a different site symmetric environment causes slight deviations from the $g$-factor around $g \sim 2$. 

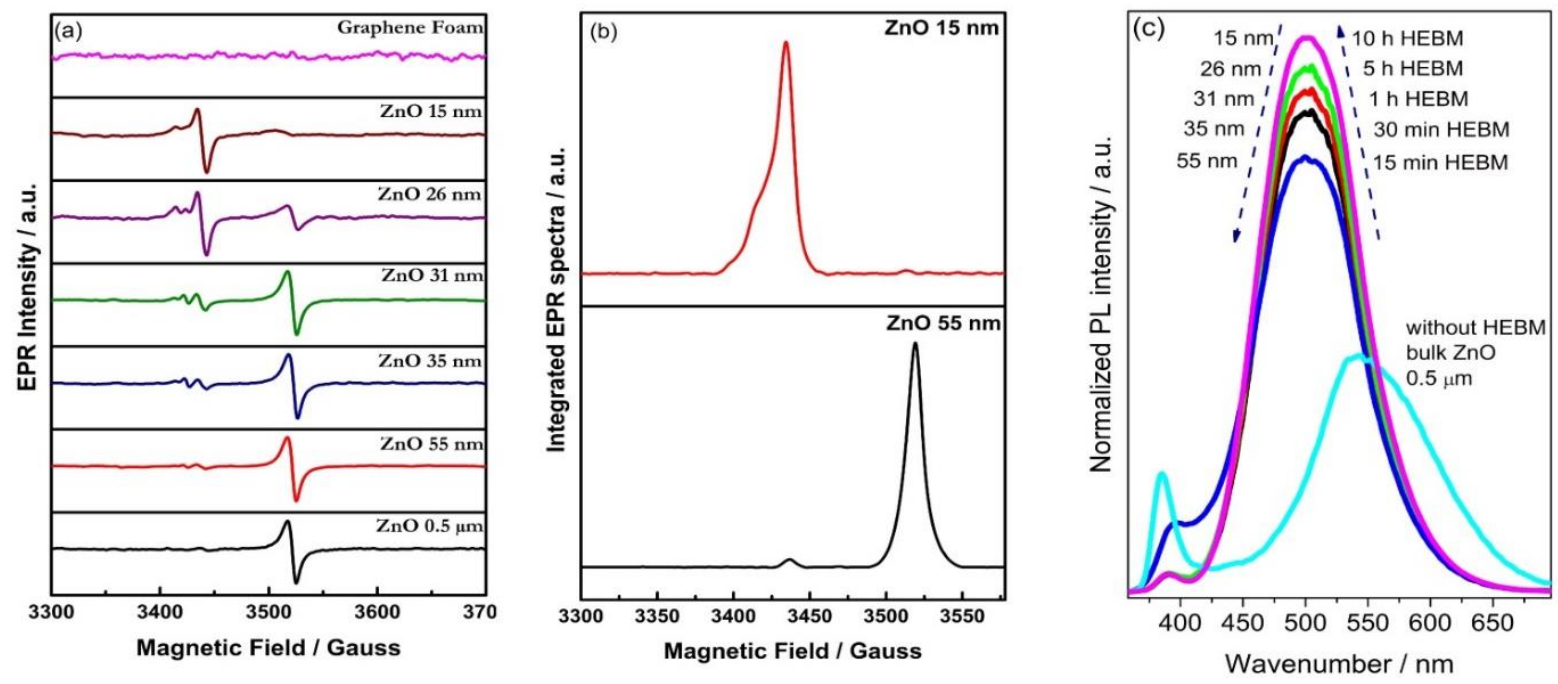

Figure 18. a) EPR spectra of HEBM ZnO nanoparticles and 3D-GF, b) absorption (integration of first derivative EPR spectra) of HEBM ZnO for 15 and $55 \mathrm{~nm}$ particle size. c) Photoluminescence spectra of HEBM ZnO nanoparticles (evolution of PL spectra with respect to crystalline size).

Several surface defects have been generally observed via EPR in particular for HEBM ZnO nanomaterials. The complete set of spectral parameters for all synthesized samples with respect to their observed $g$-factors and line width were listed in Table 2. The systematical increase was observed in line width which leads to inhomogeneous Gaussian broadening for the EPR signals stems from the core defects. Since core defects are closely related to the lattice such broadening at smaller sizes indicates strong distortions from the Wurzite hexagonal structure. Moreover, by the aid of the spin counting procedure, the defect concentrations were deduced and tabulated in Table 3. Thanks to the spin counting module of the new generation EMX Nano EPR spectrometer it is easy to get the concentration of defect states analytically. This module enables a direct comparison of the measured sample and the standard reference sample and gives the exact spin concentration. In Table 3, by decreasing the $\mathrm{ZnO}$ crystal size the defects located at the core dramatically reduces whereas the defects located at the surface significantly increase. This finding is also quite good agreement with the core-shell model. 
Table 2. $g$-factor and the line width values of core and surface defects of HEBM ZnO nanocrystals obtained from X-band EPR spectra at room temperature.

\begin{tabular}{|c|c|c|c|}
\hline $\begin{array}{c}\text { Mean } \\
\text { crystalline size }\end{array}$ & Defect location & $g$-factor & $\begin{array}{l}\text { Line Width } \\
\text { (G) }\end{array}$ \\
\hline \multirow[b]{2}{*}{$0.5 \mu \mathrm{m}$} & Core & 1.956 & 7.837 \\
\hline & Surface & 2.002 & 8.032 \\
\hline \multirow[b]{2}{*}{$15 \mathrm{~nm}$} & Core & 1.956 & 8.093 \\
\hline & $\begin{array}{l}\text { Surface1 } \\
\text { Surface2 } \\
\text { Surface3 }\end{array}$ & $\begin{array}{l}2.018 \\
2.012 \\
2.004\end{array}$ & $\begin{array}{l}3.758 \\
4.960 \\
8.790\end{array}$ \\
\hline \multirow[b]{2}{*}{$26 \mathrm{~nm}$} & Core & 1.956 & 8.233 \\
\hline & $\begin{array}{l}\text { Surface1 } \\
\text { Surface2 } \\
\text { Surface3 }\end{array}$ & $\begin{array}{l}2.018 \\
2.012 \\
2.004\end{array}$ & $\begin{array}{l}3.874 \\
4.816 \\
8.429\end{array}$ \\
\hline \multirow[b]{2}{*}{$31 \mathrm{~nm}$} & Core & 1.956 & 8.455 \\
\hline & $\begin{array}{l}\text { Surface1 } \\
\text { Surface2 } \\
\text { Surface3 }\end{array}$ & $\begin{array}{l}2.018 \\
2.012 \\
2.004\end{array}$ & $\begin{array}{l}3.924 \\
4.855 \\
8.415\end{array}$ \\
\hline \multirow[b]{2}{*}{$35 \mathrm{~nm}$} & Core & 1.956 & 9.816 \\
\hline & $\begin{array}{l}\text { Surface1 } \\
\text { Surface2 } \\
\text { Surface3 }\end{array}$ & $\begin{array}{l}2.017 \\
2.012 \\
2.004\end{array}$ & $\begin{array}{l}4.904 \\
3.775 \\
8.539\end{array}$ \\
\hline \multirow[b]{2}{*}{$55 \mathrm{~nm}$} & Core & 1.960 & 16.33 \\
\hline & $\begin{array}{l}\text { Surface1 } \\
\text { Surface2 }\end{array}$ & $\begin{array}{l}2.017 \\
2.004\end{array}$ & $\begin{array}{l}5.614 \\
8.616\end{array}$ \\
\hline
\end{tabular}

Table 3. The concentrations of the defect centers obtained by using the spin-counting procedure [47].

\begin{tabular}{|c|r|r|}
\hline $\begin{array}{c}\text { Mean } \\
\text { crystalline } \\
\text { size }\end{array}$ & $\begin{array}{c}\text { Core defects } \\
\left(\text { spins } / \mathbf{m m}^{3}\right)\end{array}$ & $\begin{array}{c}\text { Surface } \\
\text { defects } \\
\left(\text { spins } / \mathbf{m m}^{3}\right)\end{array}$ \\
\hline $\mathbf{0 . 5} \boldsymbol{\mu m}$ & $1.6 \times 10^{14}$ & $1.9 \times 10^{11}$ \\
\hline $\mathbf{5 5} \mathbf{~ n m}$ & $4.0 \times 10^{13}$ & $2.1 \times 10^{12}$ \\
\hline $\mathbf{3 5} \mathbf{~ n m}$ & $5.9 \times 10^{13}$ & $7.7 \times 10^{13}$ \\
\hline $\mathbf{3 1} \mathbf{~ n m}$ & $1.5 \times 10^{13}$ & $5.2 \times 10^{14}$ \\
\hline $\mathbf{2 6} \mathbf{~ n m}$ & $2.7 \times 10^{12}$ & $2.8 \times 10^{15}$ \\
\hline $\mathbf{1 5} \mathbf{~ n m}$ & $3.7 \times 10^{12}$ & $2.7 \times 10^{16}$ \\
\hline
\end{tabular}


Fig. 18c supports not only the EPR results but also the core-shell model as well as the representative PL features given in Fig. 11. According to PL results, HEBM samples revealed an increase in defect-related visible emission signal and the near band edge signal due to electron-hole recombination getting decrease by lowering the crystal size. Here it is worth noting that the unmilled bulk $(0.5 \mu \mathrm{m})$ sample has a completely different emission wavelength compare to HEBM samples. This is quite obvious and expected that due to collisions, strain and size effects the defect kinds, concentration, and localization may change in the lattice and causes a drastic shift in emission wavelength which is a significant blue shift here.

\subsubsection{Raman Characterization}

Raman spectroscopy has been used as a structural complementary technique since it enables us to distinguish the phase variants and defect densities at the molecular level. Raman spectrum of 3D-GF is given in Fig. 16b, while for revealing the differences as a function of HEBM, comparative Raman spectra of $\mathrm{ZnO}$ samples are given in Fig.14. In the Raman spectrum of 3D-GF (Fig. 11b), the peaks at $1590 \mathrm{~cm}^{-1}$ and $2700 \mathrm{~cm}^{-1}$ indicate the $\mathrm{G}$ and $2 \mathrm{D}$ bands, respectively. The absence of $\mathrm{D}$ band around $1350 \mathrm{~cm}^{-1}$, which shows the defects of foam, signifies that the CVD-grown 3D-GF is of high quality and defect-free. The structure of $\mathrm{ZnO}$ is wurtzite in which the tetrahedron of oxygen ions surrounds each $\mathrm{Zn}$ ion and vice versa and this orientation induces $A_{1}$ and $B_{1}$ mode polar symmetry along the $c$-axis $[8,65,66]$. Moreover, the twofold degenerate $\mathrm{E}$ modes occur when the atom displacement is perpendicular to the $c$-axis. The $E_{1}$ mode is polar while $E_{2}$ modes consist of $E_{2}(l o w)$ and $E_{2}$ (high) are non-polar oscillations. The $\mathrm{E}_{2}$ (high) mode mostly comes from the vibration of the $\mathrm{O}$ sublattice, while the $\mathrm{E}_{2}$ (low) mode is associated with the vibration of the $\mathrm{Zn}$ sublattice $[8,65,66] . A_{1}$ and $E_{1}$ modes split into transverse optical (TO) and longitudinal-optical (LO) phonons owing to the polarity. When the incident and scattered light are perpendicular to the c-axis, TO modes are allowed while LO phonon mode can appear that is parallel to the c- axis. Within all Raman modes, the $E_{2}$ modes are considered to be a Raman fingerprint for $\mathrm{ZnO}$. 


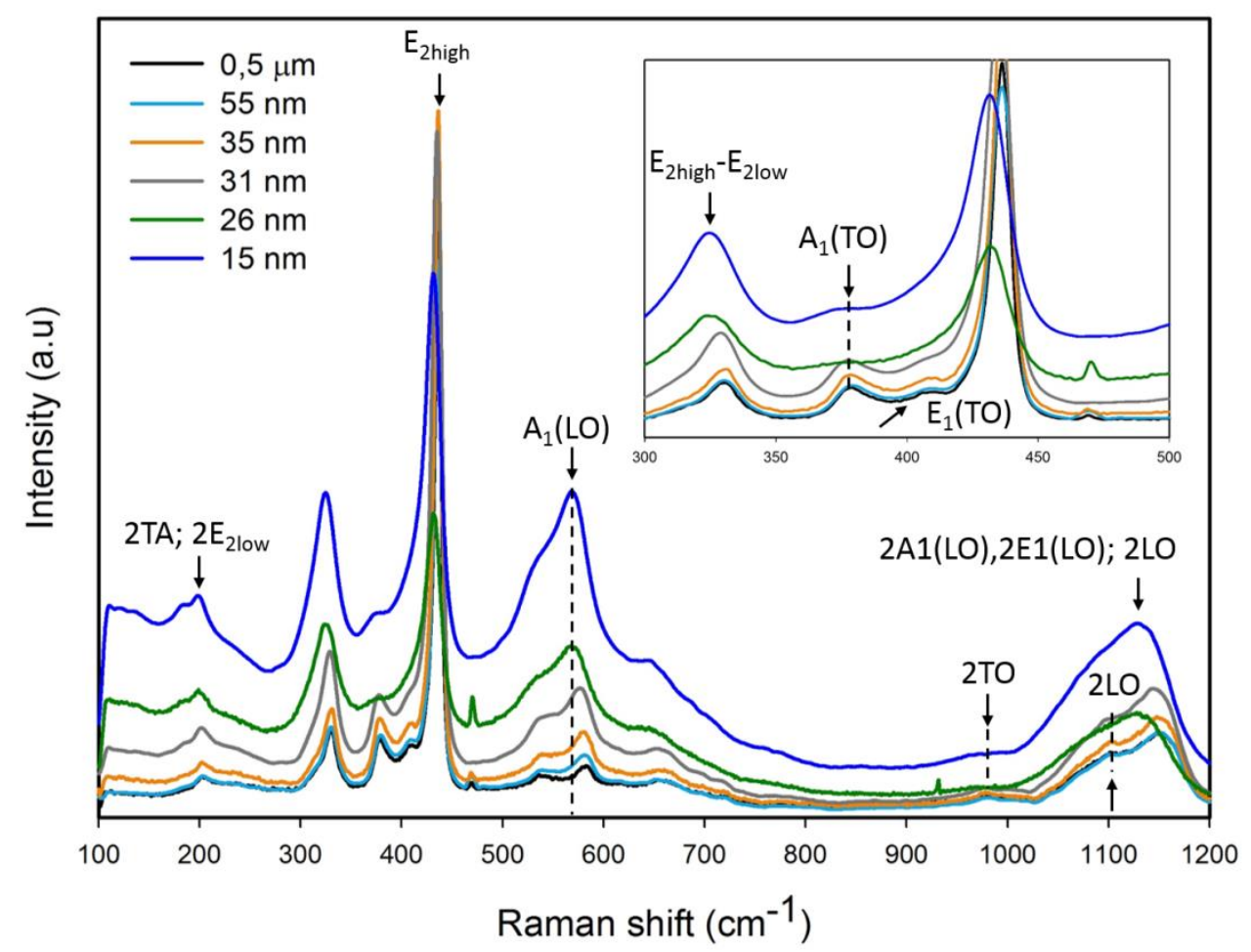

Figure 19. Size-dependent Raman spectra of HEBM ZnO crystals. The inset figure shows the region of $300-500 \mathrm{~cm}^{-1}$.

In Fig. 19, a strong peak at about $436 \mathrm{~cm}^{-1}$ belongs to the $E_{2 h i g h}$ mode which is dominantly assigned to the oxygen vibration of $\mathrm{ZnO}$. Increasing the milling time caused a shift of this peak to lower wavelengths which may be due to anisotropic internal strains, defects or impurities in the nanocrystal or optical phonon confinement due to the geometry. Since increasing the milling time affects the defect formation and thus the localization, this can be attributed to the increasing surface defect density, which is in good agreement with EPR results. The $\mathrm{E}_{2}$ (low) mode could not be detected for all $\mathrm{ZnO}$ samples that were may be due to the broad and low-intensity characteristic of this mode. However, the vibration at around $330 \mathrm{~cm}^{-1}$ is generally attributed to the second-order Raman process and assigned to the $\mathrm{E}_{2}$ (high)- $\mathrm{E}_{2}$ (low) difference mode [65]. Similar to $E_{2}$ (high) mode, with increasing milling time, $E_{2}$ (high)$\mathrm{E}_{2}$ (low) difference mode also shifted to lower wavelengths. It should be considered that the possible defects in the structure may result in second-order Raman vibrations which might be another indication of the existence of the structural defects. The peak at around $378 \mathrm{~cm}^{-1}$ is attributed to $A_{1}$ (TO) mode, while the weak shoulder peak at $410 \mathrm{~cm}^{-1}$ is assigned to $E_{1}(\mathrm{TO})$ mode. The intensities of both $A_{1}(T O)$ and $E_{1}(T O)$ modes were decreased with the decreasing particle size and $E_{1}(T O)$ peaks 
disappeared after $1 \mathrm{~h}$ of milling. This disappearance should be caused by a change in the lattice constant after milling which was previously reported in the relevant literature. The multiphoton process was detected in the high-frequency range and the most prominent bands correspond to LO overtones and its combinations [66]. The broad band at around $1150 \mathrm{~cm}^{-1}$ may correspond to 2LO scattering possibly in combination with the $2 \mathrm{~A}_{1}(\mathrm{LO})$ and $2 \mathrm{E}_{1}$ (LO) modes. This band also shifted to lower wavelengths with increasing milling time.

Table 4. First- and second-order Raman spectra frequencies of $\mathrm{ZnO}$ nanoparticles obtained via HEBM.

\begin{tabular}{|c|c|c|c|c|c|c|}
\hline & $0.5 \mu \mathrm{m}$ & $55 \mathrm{~nm}$ & $\begin{array}{c}35 \\
\mathrm{~nm}\end{array}$ & $\begin{array}{c}31 \\
\mathrm{~nm}\end{array}$ & $\begin{array}{l}26 \\
\mathrm{~nm}\end{array}$ & $15 \mathrm{~nm}$ \\
\hline Process & \multicolumn{6}{|c|}{ Raman shift $\left(\mathrm{cm}^{-1}\right)$} \\
\hline${ }^{\star 2}$ 2TA; 2E & 202 & 202 & 201 & 201 & 198 & 198 \\
\hline$E_{\text {2high- }} E_{2 l o w}$ & 330 & 330 & 330 & 328 & 324 & 324 \\
\hline$A_{1}(T O)$ & 378 & 378 & 378 & 376 & 376 & 372 \\
\hline $\mathrm{E}_{1}(\mathrm{TO})$ & 408 & 408 & 406 & ND & ND & ND \\
\hline$E_{\text {2high }}$ & 436 & 436 & 436 & 435 & 431 & 431 \\
\hline $2 B_{1 l o w} ; 2 L A$ & 537 & 536 & 536 & 535 & 534 & 533 \\
\hline$A_{1}(L O)$ & 582 & 581 & 580 & 577 & 569 & 569 \\
\hline 2то & 980 & 980 & 979 & 978 & 972 & 970 \\
\hline 2LO & 1101 & 1101 & 1100 & 1096 & ND & ND \\
\hline $\begin{array}{c}{ }^{* *} 2 A_{1}(L O), 2 E_{1}(L O) ; \\
2 L O\end{array}$ & 1154 & 1154 & 1151 & 1145 & 1129 & 1126 \\
\hline
\end{tabular}

*L, M, $\mathrm{H} ; \Gamma$ Brillouin zone points/lines [65]

${ }^{* *} \Gamma$; A-L-M Brillouin zone points/lines [65]

The bandgap of the HEBM $\mathrm{ZnO}$ nanocrystals was measured by the use of reflection spectra that were achieved by UV-Vis spectroscopy. For measuring the bandgap, the absorption coefficient $\alpha$ is obtained by the following equation, where $R_{\max }$ is the value 
of the maximum reflectance, $R_{\min }$ is the value of the minimum reflectance and $R$ is the reflectance values.

$$
\alpha=\ln \left[\frac{R_{\max }-R_{\min }}{R-R_{\min }}\right]
$$

Then in this approach typically a graph between $(\alpha h u)^{2}$ versus photon energy $(h u)$ is plotted. The intercept of this plot on the energy axis is the band gap of $\mathrm{ZnO}$ nanocrystals [67].

Table 5. Examination of bandgap energies by UV-Vis spectroscopy of HEBM ZnO nanocrystals. The band-gap values were obtained by the aid of Tauc-plot.

\begin{tabular}{|c|c|}
\hline $\begin{array}{c}\text { ZnO mean crystalline } \\
\text { size }\end{array}$ & Band Gap (eV) \\
\hline $\mathbf{5 5} \mathbf{~ n m}$ & 3.229 \\
\hline $\mathbf{3 5} \mathbf{~ n m}$ & 3.245 \\
\hline $\mathbf{3 1} \mathbf{~ n m}$ & 3.245 \\
\hline $\mathbf{2 6} \mathbf{~ n m}$ & 3.225 \\
\hline $\mathbf{1 5} \mathbf{~ n m}$ & 3.166 \\
\hline
\end{tabular}

Hence, data obtained from UV-Vis spectroscopy, interpreted by the method according to Tauc formula [68] delivering the bandgap energies are summarized in Table 4 and prove a significant decrease of the bandgap energy due to quantum confinement effect. Note that, $E_{\mathrm{g}}$ for bulk $\mathrm{ZnO}$ is $3.37 \mathrm{eV}$ at rt [31]. It is important to underline here, that the UV-Vis investigations have been done on reaction solution samples which are $3 \mathrm{~h}$ waited under the ultrasonic bath. Once the $E_{\mathrm{g}}$ is decreased dramatically from bulk to nanosize then from $55 \mathrm{~nm}$ to $15 \mathrm{~nm}$ the $E_{g}$ values deviate or saturates around the values of 3.1 and 3.2. Meaning that the limit semiconductor energy $\left(E_{g}\right)$ for HEBM samples has been reached and further engineering of band gap may not possible by decreasing the size which is also not necessary at this stage. 


\subsection{Electrochemical analysis}

To test the electrochemical performance of $\mathrm{ZnO}$ nanocrystalline electrodes we designed the supercapacitor devices by keeping separator, 3D-GF, and electrolyte unchanged in all three devices. Only the electrode with $\mathrm{ZnO}$ was changed with respect to its size as bulk $(0.5 \mu \mathrm{m}), 31 \mathrm{~nm}$ and $15 \mathrm{~nm}$. This kind of designing approach enables us not only to monitor the effects of the crystal size in the device performance but also the effect of surface defects at the nanoscale. Fig. 20a and 20b were obtained from electrochemical impedance spectroscopy (EIS) measurements presenting the results of well-known Nyquist and bode plot, respectively. By the aid of Nyquist data, the first semicircle will give the charge transfer resistance $R_{\mathrm{ct}}$. The semicircle which gives the $R_{\text {ct }}$ value of $165 \Omega$ only obtained clearly in the device where the $15 \mathrm{~nm} \mathrm{ZnO}$ nanocrystalline electrodes were used. This is a great enhancement of $\mathrm{ZnO}$ materials while as a metal oxide alone it is highly resistive up to Giga $\Omega$. But in the device together with the 3D-GF, and the electrolyte, the device itself gives a good conductive property. This is most probably the beauty of the synergy between the materials and the smart design which enables us to perform four-point electrical measurements. In the other two devices, the Warburg element and equivalent series resistance (ESR) effect is dominating the electrochemical impedance values. Interestingly, Warburg and ESR have almost no effect on $15 \mathrm{~nm}$ crystalline sizes which is indeed resistive features for the metal oxides. This can be related to the enhancement of surface defects where such defects may contribute easily to the conduction of the whole system thus its capacitance. A similar effect is visible in the Bode plot where frequency dependency was plotted with respect to the absolute value of impedance both in the logarithmic scale. Indeed, in general both Nyquist and Bode plots contain the same information since they were extracted from the same measurement data. Of course, it is always good to see how the device reacts with the frequency changes as well. The flat regions of Bode plot are always favorable for an energy system. 

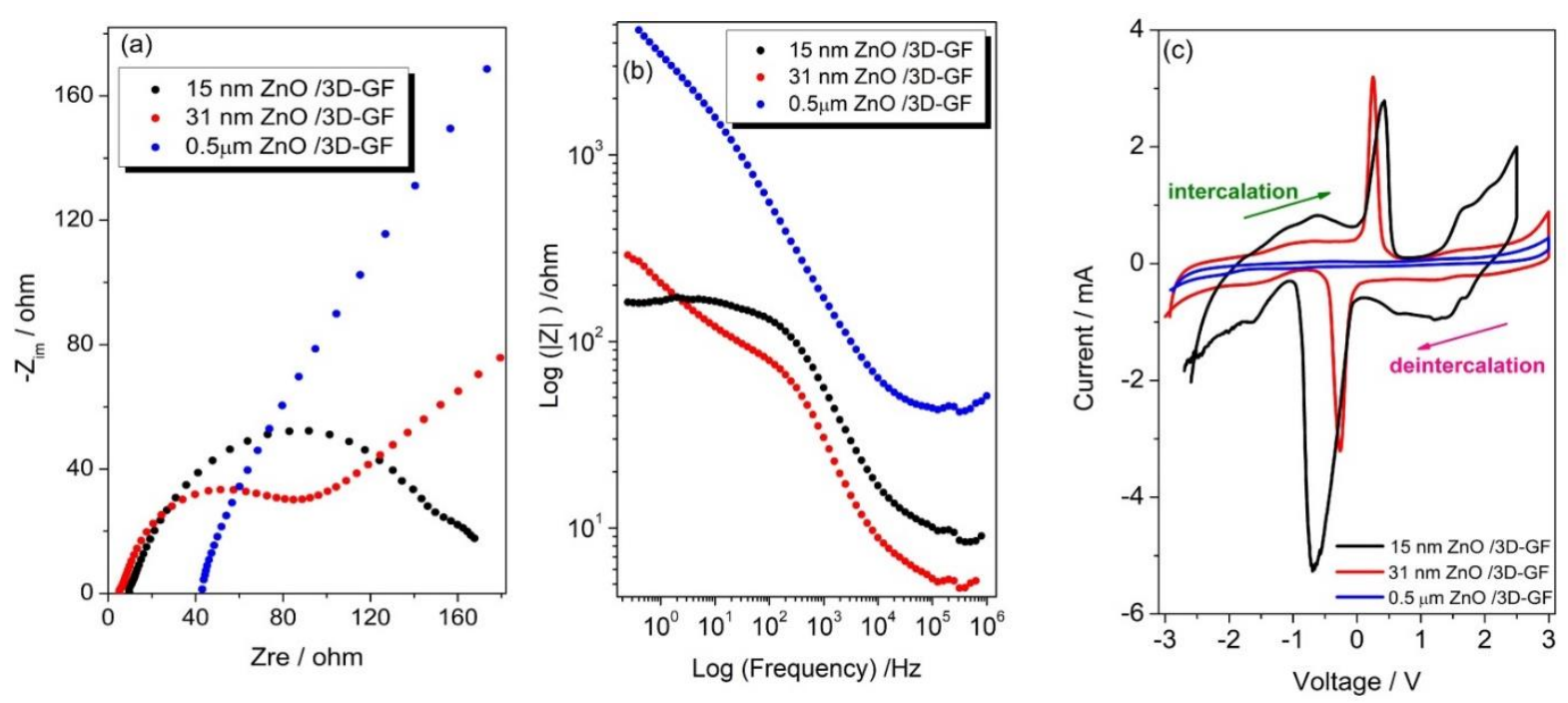

Figure 20. The electrochemical behavior of three produced asymmetric supercapacitors investigated by a) EIS in Nyquist plot of the hybrid supercapacitor, b) the variation of characteristic impedance (absolute value $|Z|)$ with frequency (Bode-plot) and, c) behavior of intercalation-deintercalation (charge-discharge) via the cyclic voltammetry (CV).

As is seen in Fig. 20b there are strong deviations from the flattening at lower frequencies. This also reflects itself strong increase in resistance values. So that such devices can optimum work around $1-10 \mathrm{kHz}$. It is worthy to say that an obvious decrease in resistance values was observed in nanosized $\mathrm{ZnO}$ compare to bulk counterpart. Finally, cyclovoltammetry (CV) measurements were applied to monitor weather the supercapacitor system work as an electric double-layer capacitor (EDCL) or Faradaic mechanism and how this mechanism depends on the size during intercalation and deintercalation period. The humps in CV plots indicate the domination of Faradaic reactions. That means the device fabricated from the bulk $\mathrm{ZnO}$ has charge storage mechanism of EDLC type whereas by decreasing the crystalline size of $\mathrm{ZnO}$ the humps arise due to the increase of surface defects. These defects create extra paths for the charges thus increase the capacity as well. The device produced from the $15 \mathrm{~nm} \mathrm{ZnO}$ has the maximum amount of humps revealing Faradaic behavior. The electrochemical performance results in both EIS and CV measurements are good agreement with the EPR and PL results. The existence of surface defects always creates extra peaks in all of these four different methods.

Reducing the crystalline size of $\mathrm{ZnO}$ causes the concentration rise of surface defects, in turn, EPR spectra have extra signal at $g \sim 2$, PL spectra has extra emission signal at the visible range, Nyquist plot revealed nice semi-circle, and finally, CV plot 
revealed extra humps during charge and discharge processes. Therefore, one may conclude that the nanosized $\mathrm{ZnO}$ has great advantages over bulky samples in terms of device performance. This can be only achieved by the extensive controlling of point defects in the system which requires advanced spectroscopic techniques such as EPR, PL, Raman, and UV-Vis as well as good electrochemical performance tests. Besides, real device production is necessary to get reliable data on the performance and synergy of all components of the supercapacitor. 
Chapter 4

\section{Conclusion}


Fossile fuels which is a main energy sources these days, has alot of side effects that are not ignorable. Supercapacitors are good alternative and candidate as a clean energy source. Although, they have many outstanding properties, thier low energy density is such a big disadvantageous. Therefore, the main The main goal of this research was to prepare a supercapcitor which has both high energy and power density. When standard structural characterization techniques of materials meet with the advanced analytical techniques for understanding electronic properties eventually one can get complete information about the material system. Systematical sizereduction thus systematic changes both in structure, defect, and electronic state can be controlled and understood. This will give an extended advantage to use such materials in the application of devices. In this work, semiconductor $\mathrm{ZnO}$ material and GF has been used for the utilization of asymmetric supercapacitor devices. The electrochemical performance of the supercapacitor devices has been tested with respect to the size of $\mathrm{ZnO}$ thus the concentration of defect centers either located on surface or core. For sure such defects regardless they are vacancies or interstitials certainly play a crucial role in the performance of the device. Here the device which has an electrode of $\mathrm{ZnO}$ with the crystalline size of $15 \mathrm{~nm}$ showed the least resistivity. Also at nanoscale metal oxide electrode revealed a Faradaic reaction mechanism having high humps in the $\mathrm{CV}$ plot. On the other hand, since the sample sizes are lying below $50 \mathrm{~nm}$ the change in bandgap is not as significant as in the drop of size from microscale to nanoscale. PL spectroscopy from the point of its selection rules for electronic transitions gives a broad emission band-type signal with peak point around green light due to the trapping of electrons or holes at the defect states. This emission signal covers all kinds of defects that can exist in $\mathrm{ZnO}$. Thanks to selection rules and resonance condition of EPR technique that from EPR spectra depending on the magnitude of spin-orbit coupling there will be strong deviations in the signal position of differently located defect centers in $\mathrm{ZnO}$ lattice. Thus it will enable us to distinguish safely different defect kinds in particular located at the surface and the core. In addition, thanks to the spin counting procedure that it is even possible to count the defect concentration or spins by just doubly integrating the first derivative EPR spectra. Accordingly here HEBM ZnO samples revealed a significant 
rise of surface defects once the crystalline size drops to a few tens of nanometer scale and of course this reflected a decrease in core defects. For future researches, it is better to further increase the ball milling time in order to find an optimum size of the $\mathrm{ZnO}$ nanoparticles. As a result, to achieve higher and better capacity with lower resistance than $165 \Omega$. In this way, these supercapacitors can be used in electrical vehicles, computer chips and devices in order to get the best performance. 


\section{References}

1. $\mathrm{Yu}, \mathrm{Z}$., et al., Supercapacitor electrode materials: nanostructures from 0 to 3 dimensions. Energy \& Environmental Science, 2015. 8(3): p. 702-730.

2. González, A., et al., Review on supercapacitors: technologies and materials. Renewable and Sustainable Energy Reviews, 2016. 58: p. 1189-1206.

3. Halper, M.S. and J.C. Ellenbogen, Supercapacitors: A brief overview. MITRE nanosystems Group, 2006.

4. Sutphin, D.M. and J.D. Bliss, Disseminated flake graphite and amorphous graphite deposit types. An analysis using grade and tonnage models. CIM Bulletin, 1990. 83(940): p. 85-89.

5. Halper, M.S. and J.C. Ellenbogen, Supercapacitors: $A$ brief overview. The MITRE Corporation, McLean, Virginia, USA, 2006: p. 1-34.

6. Hanaor, D., et al., The effects of firing conditions on the properties of electrophoretically deposited titanium dioxide films on graphite substrates. Journal of the European Ceramic Society, 2011. 31(15): p. 2877-2885.

7. Long, Y., et al., Oxidation of SO 2 to SO 3 catalyzed by graphene oxide foams. Journal of Materials Chemistry, 2011. 21(36): p. 13934-13941.

8. He, Y., et al., An environmentally friendly method for the fabrication of reduced graphene oxide foam with a super oil absorption capacity. Journal of hazardous materials, 2013. 260: p. 796-805.

9. Pei, S. and H.-M. Cheng, The reduction of graphene oxide. Carbon, 2012. 50(9): p. 3210-3228.

10. Banhart, F., J. Kotakoski, and A.V. Krasheninnikov, Structural defects in graphene. ACS nano, 2010. 5(1): p. 26--41.

11. Lehtinen, P., et al., Magnetic properties and diffusion of adatoms on a graphene sheet. Physical review letters, 2003. 91(1): p. 017202.

12. Malard, L., et al., Raman spectroscopy in graphene. Physics reports, 2009. 473(5-6): p. 51-87.

13. Saito, R., et al., Raman spectroscopy of graphene and carbon nanotubes. Advances in Physics, 2011. 60(3): p. 413-550.

14. Casiraghi, C., et al., Raman spectroscopy of graphene edges. Nano letters, 2009. 9(4): p. 1433-1441.

15. Ferrari, A.C., Raman spectroscopy of graphene and graphite: disorder, electron--phonon coupling, doping and nonadiabatic effects. Solid state communications, 2007. 143(1-2): p. 4757.

16. Ferrari, A.C., Raman spectroscopy of graphene and graphite: Disorder, electron-phonon coupling, doping and nonadiabatic effects. Solid state communications, 2007. 143(1-2): p. 4757.

17. Kasap, S., M.B. Acar, and D. Çakıroğlu, Optimization of CVD parameters on 3D graphene foam structures with response surface methodology (RSM). Materials Research Express, 2019. 6(9): p. 095604.

18. Beidaghi, M., et al., Electrostatic spray deposition of graphene nanoplatelets for high-power thin-film supercapacitor electrodes. Journal of Solid State Electrochemistry, 2012. 16(10): p. 3341-3348.

19. Wu, Z.S., et al., Field emission of single-layer graphene films prepared by electrophoretic deposition. Advanced Materials, 2009. 21(17): p. 1756-1760.

20. Kasap, S., et al., Superbat: battery-like supercapacitor utilized by graphene foam and zinc oxide (ZnO) electrodes induced by structural defects. Nanoscale Advances, 2019. 
21. Li, D., et al., Processable aqueous dispersions of graphene nanosheets. Nature nanotechnology, 2008. 3(2): p. 101.

22. Nardecchia, S., et al., Three dimensional macroporous architectures and aerogels built of carbon nanotubes and/or graphene: synthesis and applications. Chemical Society Reviews, 2013. 42(2): p. 794-830.

23. Chen, $\mathrm{H}$., et al., Mechanically strong, electrically conductive, and biocompatible graphene paper. Advanced Materials, 2008. 20(18): p. 3557-3561.

24. Pumera, M., Graphene-based nanomaterials and their electrochemistry. Chemical Society Reviews, 2010. 39(11): p. 4146-4157.

25. Zhang, Y., et al., Progress of electrochemical capacitor electrode materials: A review. International journal of hydrogen energy, 2009. 34(11): p. 4889-4899.

26. Wang, G., L. Zhang, and J. Zhang, A review of electrode materials for electrochemical supercapacitors. Chemical Society Reviews, 2012. 41(2): p. 797-828.

27. Wu, Z., et al., Graphene/metal oxide composite electrode materials for energy storage, Nano Energ. 1 (2012) 107-131.

28. Ando, K., Seeking room-temperature ferromagnetic semiconductors. Science, 2006. 312(5782): p. 1883-1885.

29. Giri, P., et al., Correlation between microstructure and optical properties of ZnO nanoparticles synthesized by ball milling. Journal of Applied Physics, 2007. 102(9): p. 093515.

30. Procek, M., T. Pustelny, and A. Stolarczyk, Influence of external gaseous environments on the electrical properties of $\mathrm{ZnO}$ nanostructures obtained by a hydrothermal method. Nanomaterials, 2016. 6(12): p. 227.

31. Erdem, E., Microwave power, temperature, atmospheric and light dependence of intrinsic defects in ZnO nanoparticles: A study of electron paramagnetic resonance (EPR) spectroscopy. Journal of alloys and compounds, 2014. 605: p. 34-44.

32. Chen, Y., et al., Plasma assisted molecular beam epitaxy of ZnO on c-plane sapphire: Growth and characterization. Journal of Applied Physics, 1998. 84(7): p. 3912-3918.

33. Najib, S. and E. Erdem, Current progress achieved in novel materials for supercapacitor electrodes: mini review. Nanoscale Advances, 2019. 1(8): p. 2817-2827.

34. Aravinda, L.S., et al., ZnO/carbon nanotube nanocomposite for high energy density supercapacitors. Electrochimica Acta, 2013. 95: p. 119-124.

35. Allen, J., Spectroscopy of lattice defects in tetrahedral II-VI compounds. Semiconductor science and technology, 1995. 10(8): p. 1049.

36. Erdem, E., Defect induced p-type conductivity in zinc oxide at high temperature: Electron paramagnetic resonance spectroscopy. Nanoscale, 2017. 9(31): p. 10983-10986.

37. Repp, S., S. Weber, and E. Erdem, Defect evolution of nonstoichiometric ZnO quantum dots. The Journal of Physical Chemistry C, 2016. 120(43): p. 25124-25130.

38. Repp, S. and E. Erdem, Controlling the exciton energy of zinc oxide ( $\mathrm{ZnO}$ ) quantum dots by changing the confinement conditions. Spectrochimica Acta Part A: Molecular and Biomolecular Spectroscopy, 2016. 152: p. 637-644.

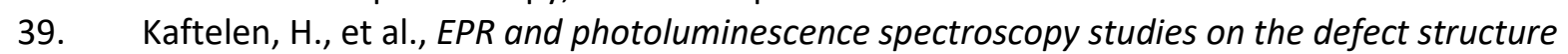
of ZnO nanocrystals. Physical Review B, 2012. 86(1): p. 014113.

40. Parashar, S., et al., Investigation of intrinsic defects in core-shell structured ZnO nanocrystals. Journal of Applied Physics, 2012. 111(11): p. 113712.

41. Jakes, P. and E. Erdem, Finite size effects in ZnO nanoparticles: An electron paramagnetic resonance (EPR) analysis. physica status solidi (RRL)-Rapid Research Letters, 2011. 5(2): p. 56-58.

42. Xia, W., et al., Improved visible-light photocurrent based on $\mathrm{ZnO} / \mathrm{ZnS}$ core-shell nanorods via interfacial engineering. Journal of Physics D: Applied Physics, 2018. 52(3): p. 035501.

43. Aravinda, L., et al., ZnO/carbon nanotube nanocomposite for high energy density supercapacitors. Electrochimica Acta, 2013. 95: p. 119-124. 
44. Chen, Y.-L., et al., Zinc oxide/reduced graphene oxide composites and electrochemical capacitance enhanced by homogeneous incorporation of reduced graphene oxide sheets in zinc oxide matrix. The Journal of Physical Chemistry C, 2011. 115(5): p. 2563-2571.

45. Peng, Z.A. and X. Peng, Formation of high-quality CdTe, CdSe, and CdS nanocrystals using CdO as precursor. Journal of the American Chemical Society, 2001. 123(1): p. 183-184.

46. Glushenkov, A., H.-Z. Zhang, and Y. Chen, Reactive ball milling to produce nanocrystalline ZnO. Materials letters, 2008. 62(24): p. 4047-4049.

47. Salah, N., et al., High-energy ball milling technique for ZnO nanoparticles as antibacterial material. International journal of nanomedicine, 2011. 6: p. 863.

48. Ullah, M., M. Ali, and S.B.A. Hamid, Surfactant-assisted ball milling: a novel route to novel materials with controlled nanostructured-a review. Reviews on Advanced Materials Science, 2014. 37.

49. Khadka, P., et al., Pharmaceutical particle technologies: An approach to improve drug solubility, dissolution and bioavailability. Asian journal of pharmaceutical sciences, 2014. 9(6): p. 304-316.

50. Tuncer, M., et al., Capacitive behaviour of nanocrystalline octacalcium phosphate (OCP)(Ca8H2 (PO4) 6.5H2O) as an electrode material for supercapacitors: biosupercaps. Nanoscale, 2019. 11(39): p. 18375-18381.

51. Genc, R., et al., High-capacitance hybrid supercapacitor based on multi-colored fluorescent carbon-dots. Scientific reports, 2017. 7(1): p. 1-13.

52. Alaş, M.Ö., et al., Feeling the power: robust supercapacitors from nanostructured conductive polymers fostered with Mn 2+ and carbon dots. Nanoscale, 2019. 11(27): p. 12804-12816.

53. Repp, S., et al., Synergetic effects of Fe 3+ doped spinel Li 4 Ti 5 O 12 nanoparticles on reduced graphene oxide for high surface electrode hybrid supercapacitors. Nanoscale, 2018. 10(4): p. 1877-1884.

54. Wolverson, D., Raman spectroscopy, in Characterization of Semiconductor Heterostructures and Nanostructures. 2008, Elsevier. p. 249-288.

55. Synetos, A. and D. Tousoulis, Invasive Imaging Techniques. Coronary Artery Disease: From Biology to Clinical Practice, 2017: p. 359.

56. Stokes, D., Principles and practice of variable pressure/environmental scanning electron microscopy (VP-ESEM). 2008: John Wiley \& Sons.

57. Speight, J.G., Environmental organic chemistry for engineers. 2016: Butterworth-Heinemann.

58. Sevilla, M.D., et al., Electron paramagnetic resonance. Vol. 19. 2007: Royal Society of Chemistry.

59. Eaton, S.S., G.R. Eaton, and L.J. Berliner, Biomedical EPR-Part A: Free radicals, metals, medicine and Physiology. 2004: Springer Science \& Business Media.

60. Orazem, M.E. A tutorial on impedance spectroscopy. in CORROSION 98. 1998. NACE International.

61. Skoog, D.A., F.J. Holler, and S.R. Crouch, Principles of instrumental analysis. 2017: Cengage learning.

62. Perkowitz, S., Optical characterization of semiconductors: infrared, Raman, and photoluminescence spectroscopy. 2012: Elsevier.

63. Schlesinger, T.E. and R.B. James, Semiconductors for room temperature nuclear detector applications. Vol. 43. 1995: Academic Press San Diego.

64. Patterson, A., The Scherrer formula for $X$-ray particle size determination. Physical review, 1939. 56(10): p. 978.

65. Schumm, M., ZnO-based semiconductors studied by Raman spectroscopy: semimagnetic alloying, doping, and nanostructures, Doctoral dissertation, Julius-Maximilians University. Dr. Diss, 2008: p. 21-23.

66. Cuscó, R., et al., Temperature dependence of Raman scattering in ZnO. Physical Review B, 2007. 75(16): p. 165202. 
67. Ruf, T., et al., Competing effects between intrinsic and extrinsic defects in pure and Mn-doped ZnO nanocrystals. Journal of Nanoparticle Research, 2016. 18(5): p. 109.

68. Tauc, J., R. Grigorovici, and A. Vancu, Optical properties and electronic structure of amorphous germanium. physica status solidi (b), 1966. 15(2): p. 627-637. 